\title{
Generalization of non-intrusive imprecise stochastic simulation for mixed uncertain variables
}

\author{
Jingwen Song ${ }^{a, d}$, Pengfei Wei ${ }^{b, *, d}$, Marcos Valdebenito ${ }^{c}$, Sifeng $\mathrm{Bi}^{d}$, Matteo Broggi ${ }^{d}$, Michael Beer ${ }^{d, e, f}$, \\ Zuxiang Lei $^{g}$ \\ a School of Aeronautics, Northwestern Polytechnical University, Xi'an 710072, China \\ ${ }^{b}$ School of Mechanics, Civil Engineering and Architecture, Northwestern Polytechnical \\ University, Xi'an 710072, China \\ ${ }^{c}$ Departmento de Obras Civiles, Universidad Tecnica Federico Santa Maria, Av. España 1680, \\ Valparaiso, Chile \\ ${ }^{d}$ Institute for Risk and Reliability, Leibniz Universität Hannover, Callinstr. 34, Hannover, \\ Germany \\ ${ }^{e}$ Institute for Risk and Uncertainty, University of Liverpool, Peach Street, L69 7ZF Liverpool, \\ United Kingdom \\ f International Joint Research Center for Engineering Reliability and Stochastic Mechanics, \\ Tongji University, Shanghai 200092, China \\ ${ }^{g}$ Institute of Geotechnical Engineering, School of Civil Engineering and Architecture, East China \\ Jiaotong University, Nanchang 330013, China
}

\begin{abstract}
Non-intrusive Imprecise Stochastic Simulation (NISS) is a recently developed general methodological framework for efficiently propagating the imprecise probability models and for estimating the resultant failure probability functions and bounds. Due to the simplicity, high efficiency, stability and good convergence, it has been proved to be one of the most appealing forward uncertainty quantification methods. However, the current version of NISS is only applicable for model with input variables characterized by precise and imprecise probability models. In real-world applications, the uncertainties of model inputs may also be characterized by non-probabilistic models such as interval model due to the extreme scarcity or imprecise information. In this paper, the NISS method is generalized for models with three kinds of mixed inputs characterized by precise probability model, non-probabilistic models and imprecise probability models respectively, and specifically, the interval model and distributional $p$-box model are exemplified. This generalization is realized by combining Bayes rule and the global NISS method, and is shown to conserve all the advantages of the classical NISS method. With this generalization, the three kinds of inputs can be propagated with only one set of function evaluations in a pure simulation manner, and two kinds of potential estimation errors are properly addressed by sensitivity indices and bootstrap. A numerical test example and the NASA uncertainty quantification challenging problem are solved to demonstrate the effectiveness of the generalized NISS procedure.
\end{abstract}

Keywords: Non-intrusive imprecise stochastic simulation; Uncertainty quantification; Non-probabilistic; Imprecise probability; Sensitivity; Bayes rule; Interval model; Bootstrap

\section{Introduction}

Uncertainty quantification (UQ) has been widely accepted as an important task in a variety of

\footnotetext{
* Corresponding author: pengfeiwei@nwpu.edu.cn
} 
research and engineering fields. For example, in the analysis and design of large civil engineering systems, the uncertainties presented in system excitations (e.g., caused by natural disasters such as earthquake and flood), material properties, degradation process modeling, etc., are quite substantial, and have to be carefully treated. Commonly, there are two kinds of uncertainties, i.e., aleatory uncertainty and epistemic uncertainty [1], while the former one is due to the intrinsic random property of parameters or events, thus cannot be reduced by collecting more information, and the later one is caused by the incompleteness of knowledge, and can be reduced by further collecting information. The above two kinds of uncertainties may appear alone, but in most real-world applications, may occur simultaneously. Characterization of the above two kinds of uncertainties with mathematical models is the first key problem of UQ.

Generally, three groups of uncertainty characterization models have been developed, i.e., the precise probability model, the non-probabilistic models [2][3], and the imprecise probability models [4]. The precise probability model (Category I) is the most classical uncertainty model, and is commonly used for characterizing the aleatory uncertainty, which usually requires a large number of data of good quality. The non-probabilistic models (Category II), including interval/convex model, fuzzy set theory, etc., can be especially useful for characterizing the imprecision of constant-but-unknown variables or for situations that the available data for random variables is extremely scarce/incomplete/imprecise [3]. As the level of knowledge increases, the interval model will degrade into its true value. The imprecise probability models (Category III), such as probability-box (p-box), evidence theory and fuzzy probability model, can be regarded as the combination of the former two kinds of models, and can be especially useful for separately characterizing the two kinds of uncertainty in a unified model framework [4]. As the volume of available information increases, the category III model will shrink to the true cumulative distribution function (CDF). The roles of three categories of characterization models are shown in Figure 1.

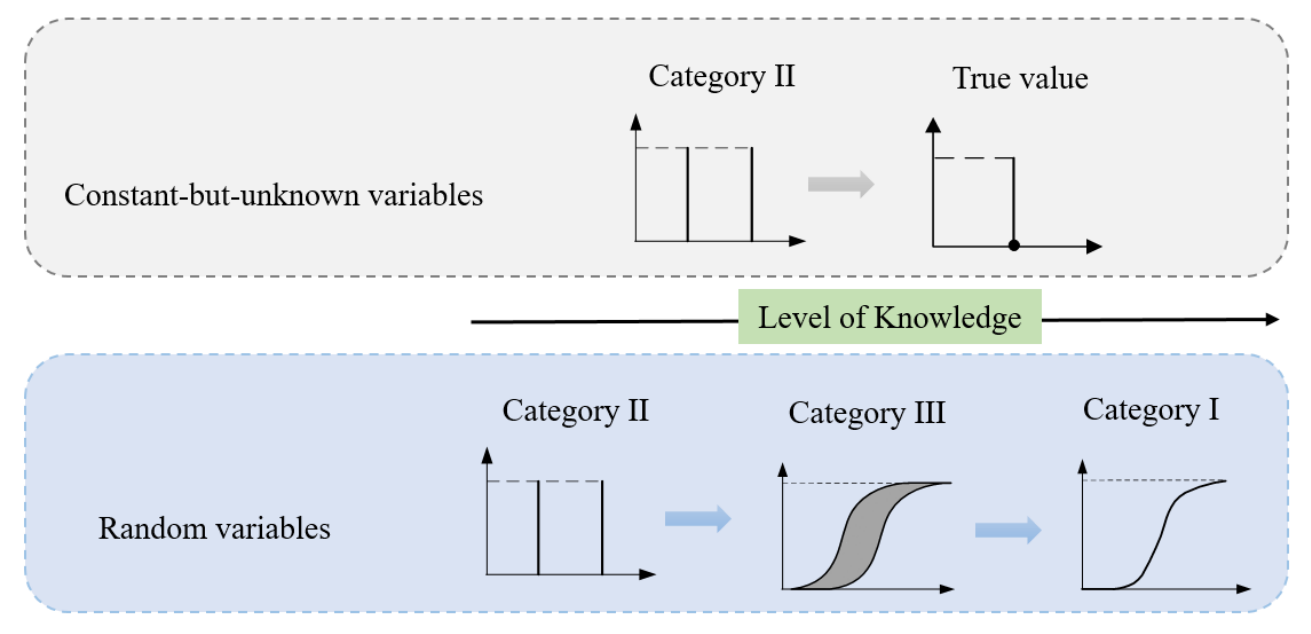

Figure 1. The roles of the three categories of characterization models in UQ.

The second key problem is the propagation of the characterization models through the computational models so as to quantify the uncertainties of the model responses, and to assess the reliability of the systems. This has been a quite big challenge especially when all the three categories of 
characterization models are present as model inputs. For example, in the NASA multidisciplinary Langley UQ challenge problem [5], the subproblem of uncertainty propagations involves 21 inputs variables characterized by the three categories of models. Propagation of the category I model has been widely studied, and a plenty of methods, such as probability density evaluation [6], importance sampling [7], subset simulation [8], line sampling [9] and active learning based surrogate model methods [10], have been developed, and shown to be effective for real-world applications. The propagation of Category II models has also been studied, and the current methods are mainly driven by optimization algorithms [2][3], which are commonly computationally expensive especially for problems with non-convex response functions and high-dimensional inputs.

The propagation of category III models is generally a double-loop process, and several strategies have been developed. The simplest procedure is to perform optimization for distribution parameters in the outer loop and then propagate the degraded category I model in the inner loop with, e.g., precise stochastic simulation method [11]. This strategy has been applied to the NASA Langley challenge problem with the utilization of genetic algorithm for outer loop optimization and Monte Carlo simulation for the inner loop analysis [12]. The second strategy is based on sampling in the outer loop so as to generate a set of interval samples for input variables, and then propagate each interval sample in the inner loop by, e.g., interval finite element analysis or optimization algorithms [13]-[15]. This strategy has been recently extended to problems with spatial/time-variant inputs [16]-[18]. The third strategy involves generating the a set of samples for input variables, and then estimating the performance values (e.g., failure probability) w.r.t. different values of the distribution parameters of category III models based on a weighting scheme. This strategy, termed as "Extended Monte Carlo simulation”, was originally developed by some of the authors in Ref. [19], and was strengthened in Ref. [20]. Although being efficient, it does not perform well for problems with high-dimensional inputs. To overcome the above shortcoming, a new methodology framework, termed as "Non-intrusive Imprecise Stochastic Simulation (NISS)", has been developed in a set of companion paper [21][22], and two groups of methods, i.e., the local NISS methods and the global NISS methods, have been presented. The NISS framework owns many advantages. It is applicable for high-dimensional problems with numerical estimation errors being properly addressed, and meanwhile, provides good balance for local and global performances. Any precise stochastic simulation such as subset simulation and line sampling can be injected into this framework so as to properly address different types of problems, and the sensitivity information of the epistemic uncertainty is generated as a byproduct. Besides, the NISS framework avoids performing optimization on the model response functions, thus can properly address problems with even non-convex response functions. However, the current version of NISS is only applicable for problems with inputs characterized by category I and category III models.

The aim of this paper is to generalize NISS to the situation where all the three categories of characterization models are involved, and specifically, the interval model in category II and the distributional p-box model in category III are concerned. The generalization is realized based on Bayes rule and the global NISS method, and is shown to own all the advantages of the original NISS method. The truncation errors as well as the influential component functions are identified by the Sobol' sensitivity indices, and the statistical errors are quantified by bootstrap scheme. The proposed method is 
demonstrated by a toy test example, and is then applied to solve the reliability analysis subproblem of the NASA Langley UQ challenge problem.

The rest of this paper is organized as follows. Section 2 gives briefly reviews the NISS method, followed by the generalization of NISS in section 3. In section 4, the toy test example and the NASA Langley UQ challenge problem are introduced to demonstrate the proposed method. Section 5 gives conclusions and useful discussions.

\section{Brief review of NISS}

In this section, we briefly review the classical NISS method for propagating imprecise probability models, and specifically, for distributional $p$-box model. Let $\boldsymbol{x}=\left(x_{1}, x_{2}, \ldots, x_{n}\right)^{T}$ denote the $n$ dimensional vector of random input variables with joint probability density function (PDF) $f_{\mathbf{X}}(\boldsymbol{x} \mid \boldsymbol{\theta})$, where $\boldsymbol{\theta}=\left(\theta_{1}, \theta_{2}, \ldots, \theta_{\boldsymbol{d}}\right)^{\boldsymbol{T}}$ refers to the $d$-dimensional vector of non-deterministic distribution parameters, each $\theta_{i}$ of which is assumed to be an interval parameter with support $\left[\underline{\theta}_{i}, \bar{\theta}_{i}\right]$. Let $\underline{\boldsymbol{\theta}}=$ $\left(\underline{\theta}_{1}, \underline{\theta}_{2}, \ldots, \underline{\theta}_{d}\right)^{T}$ and $\overline{\boldsymbol{\theta}}=\left(\bar{\theta}_{1}, \bar{\theta}_{2}, \ldots, \bar{\theta}_{d}\right)^{T}$. With this $p$-box model, the aleatory uncertainty of $\boldsymbol{x}$ is characterized by its joint PDF, while the epistemic uncertainty subjected to incomplete knowledge is represented by the hyper-rectangle support of $\boldsymbol{\theta}$. In real-world applications, the support of $\boldsymbol{\theta}$ can be estimated by confidence interval estimation procedure, and as the data volume of $\boldsymbol{x}$ increases, the support of $\boldsymbol{\theta}$ will shrink, indicating the reduction of epistemic uncertainty. With enough data, the support of $\boldsymbol{\theta}$ shrinks into a fixed point, and then the $p$-box model degrades into a precise probability model characterizing only aleatory uncertainty. Without loss of generality, we assume that the input variables are independent, and their joint PDF can be expressed as $f_{\mathbf{X}}(\boldsymbol{x} \mid \boldsymbol{\theta})=\prod_{i=1}^{n} f_{\mathrm{X}_{i}}\left(x_{i} \mid \boldsymbol{\theta}_{i}\right)$, where $f_{\mathrm{X}_{i}}\left(x_{i} \mid \boldsymbol{\theta}_{i}\right)$ implies the marginal PDF of $x_{i}$, and $\boldsymbol{\theta}_{i}=\left(\theta_{i 1}, \theta_{i 2}, \ldots, \theta_{d_{i}}\right)^{T}$ indicates the $d_{i}$-dimensional vector of the distribution parameters of $x_{i}$. For performing the global NISS procedure, an auxiliary PDF $f_{\boldsymbol{\Theta}}(\boldsymbol{\theta})$ should be introduced. In this paper, we assume that each $\theta_{i}$ follows independent uniform distribution within its respective support $\left[\underline{\theta}_{i}, \bar{\theta}_{i}\right]$, and the marginal PDF is denoted as $f_{\Theta_{i}}\left(\theta_{i}\right)$.

Let $g(\boldsymbol{x})$ indicate the model response function (also called limit state function for reliability analysis, or simply $g$-function) of the computational model. In this paper, only one-dimensional response is exemplified for illustrating the proposed method. We define a sub-domain of input space as $Z(z)=$ $\{\boldsymbol{x}: g(\boldsymbol{x})<z\}$, and then the indicator function corresponding to this sub-domain can be defined as $I_{Z}(x)=1$ if $\boldsymbol{x} \in Z(z)$; else $I_{Z}(x)=0$. For reliability analysis, we assume that the failure happens when the model response is less than zero. Then the failure domain can be defined as $F=Z(0)$, and the corresponding indicator function of $F$ is defined as $I_{F}(x)=1$ if $\boldsymbol{x} \in F$; else $I_{F}(\boldsymbol{x})=0$. Based on the above definition, the $\mathrm{CDF}$ of the model response w.r.t $\boldsymbol{\theta}$ can be formulated as:

$$
D_{\mathrm{Z}}(z \mid \boldsymbol{\theta})=\mathrm{E}_{\mathbf{X}}\left[I_{Z}(\boldsymbol{x})\right]=\int_{\mathbf{R}^{n}} I_{Z}(\boldsymbol{x}) f_{\mathbf{X}}(\boldsymbol{x} \mid \boldsymbol{\theta}) \mathrm{d} \boldsymbol{x}
$$

, where $E_{\mathbf{X}}[\cdot]$ indicates the expectation operator w.r.t. $\boldsymbol{x}$. The failure probability function can then be derived as $\mathrm{P}_{f}(\boldsymbol{\theta})=D_{\mathrm{Z}}(0 \mid \boldsymbol{\theta})$.

The NISS methods are developed based on high-dimensional model representation (HDMR) decomposition of $D_{\mathrm{Z}}(z \mid \boldsymbol{\theta})$ w.r.t. $\boldsymbol{\theta}$, where the local NISS methods are based on cut-HDMR decomposition and the global ones are devised from random sampling (RS)-HDMR decomposition [23]. Here only the simplest global NISS method is reviewed since only this method will be generalized. The 
RS-HDMR decomposition of $D_{\mathrm{Z}}(z \mid \boldsymbol{\theta})$ reads:

$$
D_{\mathrm{Z}}(z \mid \boldsymbol{\theta})=D_{\mathrm{Z}, 0}(z)+\sum_{1 \leq i \leq d} D_{\mathrm{Z}, \Theta_{i}}\left(z \mid \theta_{i}\right)+\sum_{1 \leq i<j \leq d} D_{\mathrm{Z}, \boldsymbol{\theta}_{i j}}\left(z \mid \boldsymbol{\theta}_{i j}\right)+\cdots+D_{\mathrm{Z}, \boldsymbol{\theta}}(z \mid \boldsymbol{\theta})
$$

, where $\boldsymbol{\theta}_{i j}=\left(\theta_{i}, \theta_{j}\right)^{T}$, and the constant component as well as the first two order component functions are formulated as:

$$
\begin{gathered}
D_{\mathrm{Z}, 0}(z)=\mathrm{E}_{\boldsymbol{\Theta}}\left[D_{\mathrm{Z}}(z \mid \boldsymbol{\theta})\right] \\
D_{\mathrm{Z}, \Theta_{i}}\left(z \mid \theta_{i}\right)=\mathrm{E}_{\boldsymbol{\Theta}_{-i}}\left[D_{\mathrm{Z}}(z \mid \boldsymbol{\theta})\right]-D_{\mathrm{Z}, 0} \\
D_{\mathrm{Z}, \boldsymbol{\Theta}_{i j}}\left(z \mid \boldsymbol{\theta}_{i j}\right)=\mathrm{E}_{\boldsymbol{\Theta}_{-i j}}\left[D_{\mathrm{Z}}(z \mid \boldsymbol{\theta})\right]-D_{\mathrm{Z}, \Theta_{i}}-D_{\mathrm{Z}, \Theta_{j}}-D_{\mathrm{Z}, 0}
\end{gathered}
$$

In Eq. (3), $\mathrm{E}_{\boldsymbol{\Theta}}[\cdot]$ indicates the expectation operator w.r.t. $\boldsymbol{\theta}, \mathrm{E}_{\boldsymbol{\Theta}_{-i}}[\cdot]$ is the expectation operator w.r.t. the $(d-1)$-dimensional vector $\boldsymbol{\theta}_{-i}$ consisting of all the elements of $\boldsymbol{\theta}$ but $\theta_{i}$, and $\mathrm{E}_{\boldsymbol{\Theta}_{-i j}}[\cdot]$ refers to the expectation operator w.r.t. $\boldsymbol{\theta}_{-i \boldsymbol{j}}$, which consists of all the components of $\boldsymbol{\theta}$ but $\boldsymbol{\theta}_{i j}$. The expectation of each component other than $D_{\mathrm{Z}, 0}$ equals to zero, and are mutually orthogonal.

Given a set of joint samples $\left\{\boldsymbol{x}^{(k)}, \boldsymbol{\theta}^{(k)}\right\}_{k=1,2, \cdots, N}$ following $f_{\mathbf{X}}(\boldsymbol{x} \mid \boldsymbol{\theta}) f_{\boldsymbol{\Theta}}(\boldsymbol{\theta})$, the NISS estimators of the RS-HDMR components are given as:

$$
\begin{gathered}
\widehat{D}_{\mathrm{Z}, 0}(\mathrm{Z})=\frac{1}{N} \sum_{k=1}^{N} I_{Z}\left(\boldsymbol{x}^{(k)}\right) \\
\widehat{D}_{\mathrm{Z}, \Theta_{i}}\left(z \mid \theta_{i}\right)=\frac{1}{N} \sum_{k=1}^{N} I_{Z}\left(\boldsymbol{x}^{(k)}\right) r_{\Theta_{i}}\left(\theta_{i} \mid \boldsymbol{x}^{(k)}, \boldsymbol{\theta}^{(k)}\right) \\
\widehat{D}_{\mathrm{Z}, \Theta_{i j}}\left(Z \mid \boldsymbol{\theta}_{i j}\right)=\frac{1}{N} \sum_{k=1}^{N} I_{Z}\left(\boldsymbol{x}^{(k)}\right) r_{\boldsymbol{\Theta}_{i j}}\left(\boldsymbol{\theta}_{i j} \mid \boldsymbol{x}^{(k)}, \boldsymbol{\theta}^{(k)}\right)
\end{gathered}
$$

, where

$$
\begin{gathered}
r_{\Theta_{i}}\left(\theta_{i} \mid \boldsymbol{x}^{(k)}, \boldsymbol{\theta}^{(k)}\right)=\frac{f_{\mathbf{X}}\left(\boldsymbol{x}^{(k)} \mid \theta_{i}, \boldsymbol{\theta}_{-i}^{(k)}\right)}{f_{\mathbf{X}}\left(\boldsymbol{x}^{(k)} \mid \boldsymbol{\theta}^{(k)}\right)}-1 \\
r_{\boldsymbol{\theta}_{i j}}\left(\boldsymbol{\theta}_{i j} \mid \boldsymbol{x}^{(k)}, \boldsymbol{\theta}^{(k)}\right)=\frac{f_{\mathbf{X}}\left(\boldsymbol{x}^{(k)} \mid \boldsymbol{\theta}_{i j}, \boldsymbol{\theta}_{-i j}^{(k)}\right)}{f_{\mathbf{X}}\left(\boldsymbol{x}^{(k)} \mid \boldsymbol{\theta}^{(k)}\right)}-\frac{f_{\mathbf{X}}\left(\boldsymbol{x}^{(k)} \mid \theta_{i}, \boldsymbol{\theta}_{-i}^{(k)}\right)}{f_{\mathbf{X}}\left(\boldsymbol{x}^{(k)} \mid \boldsymbol{\theta}^{(k)}\right)}-\frac{f_{\mathbf{X}}\left(\boldsymbol{x}^{(k)} \mid \theta_{j}, \boldsymbol{\theta}_{-j}^{(k)}\right)}{f_{\mathbf{X}}\left(\boldsymbol{x}^{(k)} \mid \boldsymbol{\theta}^{(k)}\right)}+1
\end{gathered}
$$

The above estimators are all unbiased, and their variances can be easily derived, as shown in Ref. [21]. One should note that the ratio function in Eq. (5) are derived based on the assumption that the auxiliary distributions of $\boldsymbol{\theta}$ are all uniform. If non-uniform distribution is assumed, the ratio functions are still made of density functions, but the formulations will be different.

The accuracy of NISS may also be affected by the RS-HDMR truncation, and as indicated in Refs. [21] and [24], the truncation errors can be subtly assessed by the Sobol' sensitivity indices. Due to the orthogonality of the RS-HDMR component functions, taking variance to both side of Eq. (2) yields [24][25]:

$$
\operatorname{var}\left[D_{\mathrm{Z}}(\mathrm{z} \mid \boldsymbol{\theta})\right]=\sum_{1 \leq i \leq d} \operatorname{var}\left[D_{\mathrm{Z}, \Theta_{i}}\left(z \mid \theta_{i}\right)\right]+\sum_{1 \leq i<j \leq d} \operatorname{var}\left[D_{\mathrm{Z}, \boldsymbol{\Theta}_{i j}}\left(\mathrm{z} \mid \boldsymbol{\theta}_{i j}\right)\right]+\cdots+\operatorname{var}\left[D_{\mathrm{Z}, \boldsymbol{\Theta}}(z \mid \boldsymbol{\theta})\right]
$$

Based on the above variance decomposition, the Sobol' sensitivity index can be defined for each component function. For the first- and second-order component functions, the Sobol' indices are defined as [25][26]:

$$
S_{\Theta_{i}}=\frac{\operatorname{var}\left[D_{\left.\mathrm{Z}, \Theta_{i}\left(z \mid \theta_{i}\right)\right]}\right.}{\operatorname{var}\left[D_{\mathrm{Z}}(\mathrm{Z} \mid \boldsymbol{\theta})\right]}
$$

and

$$
S_{\boldsymbol{\Theta}_{i j}}=\frac{\operatorname{var}\left[D_{\mathrm{Z}, \boldsymbol{\Theta}_{i j}}\left(z \mid \boldsymbol{\theta}_{i j}\right)\right]}{\operatorname{var}\left[D_{\mathrm{Z}}(Z \mid \boldsymbol{\theta})\right]}
$$


The Sobol' indices for higher order component functions can be similarly defined. These sensitivity indices can be easily computed by numerically integrating the NISS estimators in Eq. (4). In the classical global NISS procedure, the Sobol' indices are served for three purposes. As the Sobol' indices measure the relative importance of each RS-HDMR component function, the component functions with very small values of Sobol' indices can be neglected while synthesizing the estimate of failure probability function. Based on the interpretation of Sobol' indices, the first-order index $S_{i}$ measures the contribution of the epistemic uncertainty of $\theta_{i}$ to the epistemic uncertainty of response CDF, and the second-order index $S_{\boldsymbol{\Theta}_{i j}}$ measures the second-order interaction contribution between $\theta_{i}$ and $\theta_{j}$. Thereof, the Sobol' indices can also be used for identifying the main sources of epistemic uncertainty present in model response CDF, thus can be especially useful for further collecting information and for specifying the important parameters to be calibrated in inverse uncertainty quantification. Besides, $1-\sum_{1 \leq i \leq d} S_{\Theta_{i}}-\sum_{1 \leq i<j \leq d} S_{\Theta_{i j}}$ measures the truncation error of the second-order RS-HDMR decomposition. If this value is less than a pre-specified threshold (say 0.03), it is asserted that the truncation error is small.

For reliability and rare event analysis, the subset simulation as well as active learning procedure have both been injected into the NISS framework, and shown to be effective and of wide applicability [22]. The main drawback of the above NISS method is the inapplicability to category II models. In the next section, we discuss the necessity of the generalization of NISS for mixed uncertain variables, and then develop a simple but effective strategy for realizing the generalization.

\section{Generalization of NISS}

\subsection{Discussions on non-probabilistic models}

Aside for the precise and imprecise probability models, several non-probabilistic models, such as interval/convex models [2][3], fuzzy set theory [2][27], and possibility theory derived from fuzzy sets [27], have also been developed for characterizing uncertainty. In this paper, we take the independent interval model of category II as an example. The non-probabilistic models are important complements to the precise and imprecise probability models in the following two situations.

- Situation 1: constant-but-unknown variable. In this situation, we know that the variable under consideration is a constant, but due to measurement error, ambiguity, subjective of expert opinion, etc., we don't know the exact value of this constant. The available information is a collection of intervals. Based on this assumption, the variable has only epistemic uncertainty, which should be characterized by the intersection of this collection of intervals under the assumption that each interval includes the true value of the variable. A typical example of this situation is the reliability analysis of existing structures. The dimension and material property parameters of an existing structures are unquestionably deterministic, but due to measurement errors, these parameters should be modeled as interval variables.

- Situation 2: random variable subjected to extreme lack of information. In this situation, the variable under consideration is a random variable, but due to the extreme lack of information, we cannot generate a proper imprecise probability model with confidence. In this situation, both aleatory and epistemic uncertainties are present, but as the epistemic uncertainty is dominant, it is better to model the variable with non-probabilistic models such as interval model. This situation often occurs in the design of future structures when new materials are utilized, and we 
have only small number of inaccurate experimental data (modeled by intervals) on the material property parameters. We can simply model the uncertainty of the parameter by the union of the collected intervals.

Otherwise, if the amount and quality of the available data for a random variable allow us to model its aleatory and epistemic uncertainties with imprecise probability models, we'd better use the imprecise probability models since they are more informative. The above two situations are schematically illustrated in Figure 1.

In practical applications, the three categories of input variables, i.e., the non-probabilistic variables, the imprecise random variables and the precise random variables, may exist simultaneously in the same analysis task, and it is necessary to extend the NISS method to such situation. In the next subsection, we present the generalization.

\subsection{The developed method}

Let denote by $\boldsymbol{y}=\left(y_{1}, y_{2}, \ldots, y_{m}\right)^{T}$ the $m$-dimensional independent interval variables with hyperrectangular support $[\underline{\boldsymbol{y}}, \overline{\boldsymbol{y}}]=\times_{i=1}^{m}\left[\underline{y}_{i}, \bar{y}_{i}\right]$, where $\times$ indicates the Cartesian product, $\left[\underline{y}_{i}, \bar{y}_{i}\right]$ is the support of $y_{i}$ with $\bar{y}_{i}>\underline{y_{i}}, \underline{\boldsymbol{y}}=\left(\underline{y}_{1}, \underline{y}_{2}, \ldots, \underline{y_{m}}\right)^{T}$ is the vector of the lower bounds, and $\overline{\boldsymbol{y}}=$ $\left(\bar{y}_{1}, \bar{y}_{2}, \ldots, \bar{y}_{m}\right)^{T}$ is the vector of upper bounds. Then, the model response function is written as $g(\boldsymbol{x}, \boldsymbol{y})$, and the indicator function $I_{Z}(\boldsymbol{x}, \boldsymbol{y})$ and $I_{F}(\boldsymbol{x}, \boldsymbol{y})$ can be similarly defined. In this section, we take the failure probability function $\mathrm{P}_{f}(\boldsymbol{\theta}, \boldsymbol{y})$ as an example to discuss the generalization of NISS. By definition, the failure probability function is formulated as:

$$
\mathrm{P}_{f}(\boldsymbol{\theta}, \boldsymbol{y})=\mathrm{E}_{\mathbf{X}}\left[I_{F}(\boldsymbol{x}, \boldsymbol{y})\right]=\int_{\mathbf{R}^{n}} I_{F}(\boldsymbol{x}, \boldsymbol{y}) f_{\mathbf{X}}(\boldsymbol{x} \mid \boldsymbol{\theta}) \mathrm{d} \boldsymbol{x}
$$

With the above setting, the epistemic uncertainties of model inputs are characterized by the hyperrectangles $\boldsymbol{\theta} \in[\underline{\boldsymbol{\theta}}, \overline{\boldsymbol{\theta}}]$ and $\boldsymbol{y} \in[\underline{\boldsymbol{y}}, \overline{\boldsymbol{y}}]$. Similarly, for implementing the global NISS method, we need to attribute an auxiliary distribution for each $y_{i}$. Without loss of generality, we assume that each $y_{i}$ follows independent uniform distribution, and denote the corresponding marginal PDF as $f_{\mathrm{Y}_{i}}\left(y_{i}\right)$, and the joint PDF as $f_{\mathbf{Y}}(\boldsymbol{y})=\prod_{i=1}^{m} f_{\mathrm{Y}_{i}}\left(y_{i}\right)$. The effects of the auxiliary distribution will be discussed later.

With RS-HDMR, the failure probability function $\mathrm{P}_{f}(\boldsymbol{\theta}, \boldsymbol{y})$ can be decomposed as:

$$
\begin{aligned}
\mathrm{P}_{f}(\boldsymbol{\theta}, \boldsymbol{y})=\mathrm{P}_{f, 0}+ & \sum_{1 \leq i \leq d} \mathrm{P}_{f, \Theta_{i}}\left(\theta_{i}\right)+\sum_{1 \leq i<j \leq d} \mathrm{P}_{f, \boldsymbol{\Theta}_{i j}}\left(\boldsymbol{\theta}_{i j}\right)+\sum_{1 \leq i \leq m} \mathrm{P}_{f, \mathrm{Y}_{i}}\left(y_{i}\right)+\sum_{1 \leq i<j \leq m} \mathrm{P}_{f, \mathbf{Y}_{i j}}\left(\boldsymbol{y}_{i j}\right) \\
& +\sum_{1 \leq i \leq d, 1 \leq j \leq m} \mathrm{P}_{f, \Theta_{i}, Y_{j}}\left(\theta_{i}, y_{j}\right)+\cdots+\mathrm{P}_{f, \boldsymbol{\Theta}, \mathrm{Y}}(\boldsymbol{\theta})
\end{aligned}
$$

where

$$
\begin{gathered}
\mathrm{P}_{f, 0}=\mathrm{E}_{\boldsymbol{\Theta}, \mathbf{Y}}\left[\mathrm{P}_{f}(\boldsymbol{\theta}, \boldsymbol{y})\right] \\
\mathrm{P}_{f, \Theta_{i}}\left(\theta_{i}\right)=\mathrm{E}_{\boldsymbol{\Theta}_{-i}, \mathbf{Y}}\left[\mathrm{P}_{f}(\boldsymbol{\theta}, \boldsymbol{y})\right]-\mathrm{P}_{f, 0} \\
\mathrm{P}_{f, \boldsymbol{\Theta}_{i j}}\left(\boldsymbol{\theta}_{i j}\right)=\mathrm{E}_{\boldsymbol{\Theta}_{-i j}, \mathbf{Y}}\left[\mathrm{P}_{f}(\boldsymbol{\theta}, \boldsymbol{y})\right]-\mathrm{P}_{f, \Theta_{i}}-\mathrm{P}_{f, \Theta_{j}}-\mathrm{P}_{f, 0} \\
\mathrm{P}_{f, Y_{i}}\left(y_{i}\right)=\mathrm{E}_{\boldsymbol{\Theta}, \mathrm{Y}_{-i}}\left[\mathrm{P}_{f}(\boldsymbol{\theta}, \boldsymbol{y})\right]-\mathrm{P}_{f, 0} \\
\mathrm{P}_{f, \mathbf{Y}_{i j}}\left(\boldsymbol{y}_{i j}\right)=\mathrm{E}_{\boldsymbol{\Theta}, \mathbf{Y}_{-i j}}\left[\mathrm{P}_{f}(\boldsymbol{\theta}, \boldsymbol{y})\right]-\mathrm{P}_{f, Y_{i}}-\mathrm{P}_{f, Y_{j}}-\mathrm{P}_{f, 0} \\
\mathrm{P}_{f, \Theta_{i}, Y_{j}}\left(\theta_{i}, y_{j}\right)=\mathrm{E}_{\boldsymbol{\Theta}_{-i}, \mathbf{Y}_{-j}}\left[\mathrm{P}_{f}(\boldsymbol{\theta}, \boldsymbol{y})\right]-\mathrm{P}_{f, \Theta_{i}}-\mathrm{P}_{f, Y_{j}}-\mathrm{P}_{f, 0}
\end{gathered}
$$

, and $\mathrm{E}_{\boldsymbol{\Theta}, \mathbf{Y}}[\cdot]$ indicates the expectation operator w.r.t. both $\boldsymbol{\theta}$ and $\boldsymbol{y}, \mathrm{E}_{\boldsymbol{\Theta}_{-i}, \mathbf{Y}}[\cdot]$ refers to the expectation 
taken w.r.t. $\boldsymbol{\theta}_{-\boldsymbol{i}}$ and $\boldsymbol{y}$, etc.

For estimating the RS-HDMR component functions in Eq. (11), we need first to generate a joint sample set $S=\left\{\left(\boldsymbol{x}^{(\boldsymbol{k})}, \boldsymbol{\theta}^{(\boldsymbol{k})}, \boldsymbol{y}^{(\boldsymbol{k})}\right)\right\}_{k=1,2, \cdots, N}$ following joint PDF $f_{\mathbf{X}}(\boldsymbol{x} \mid \boldsymbol{\theta}) f_{\boldsymbol{\Theta}}(\boldsymbol{\theta}) f_{\mathbf{Y}}(\boldsymbol{y})$. As $(\boldsymbol{x}, \boldsymbol{\theta})$ is independent of $\boldsymbol{y}$, their samples can be generated independently, and the procedure is given as follows.

- Generation of joint samples for $(\boldsymbol{x}, \boldsymbol{\theta})$. Generate a sample matrix $\mathbf{U}=$ $\left(u_{k i}\right)_{k=1, \cdots N, i=1, \cdots,(n+d)}$ with $N$ rows and $(n+d)$ columns by, e.g., Latin hypercube sampling, each column of which follows independent uniform distribution between 0 and 1 ; Then, generate the sample $\theta_{i}^{(k)}$ for each $\theta_{i}$ by $\theta_{i}^{(k)}=D_{\Theta_{i}}^{-1}\left(u_{k(n+i)}\right)$, where $D_{\Theta_{i}}^{-1}(\cdot)$ indicates the inverse CDF of $\theta_{i}$; At last, create the sample $x_{i}^{(k)}=D_{\mathrm{X}_{i}}^{-1}\left(u_{k i} \mid \boldsymbol{\theta}_{i}^{(k)}\right)$, where $D_{\mathrm{X}_{i}}^{-1}($. $\left.\mid \boldsymbol{\theta}_{i}^{(k)}\right)$ is the inverse CDF of $x_{i}$ with its distribution parameters fixed at $\boldsymbol{\theta}_{i}^{(k)}$.

- Generation of samples for $\boldsymbol{y}$. Generate a sample matrix $\mathbf{V}=\left(v_{k i}\right)_{k=1, \cdots N, i=1, \cdots, m}$, each column of which is independently and uniformly distributed between 0 and 1 ; Then, compute the sample $y_{i}^{(k)}$ for each $y_{i}$ by $y_{i}^{(k)}=D_{\mathrm{Y}_{i}}^{-1}\left(v_{k i}\right)$, where $D_{\mathrm{Y}_{i}}^{-1}(\cdot)$ is the inverse CDF of $y_{i}$.

The NISS estimators of the component functions $\mathrm{P}_{f, 0}, \mathrm{P}_{f, \Theta_{i}}\left(\theta_{i}\right)$ and $\mathrm{P}_{f, \boldsymbol{\Theta}_{i j}}\left(\boldsymbol{\theta}_{i j}\right)$ in Eq. (11) are similar to those in Eq. (4), and are formulated as:

$$
\begin{gathered}
\widehat{\mathrm{P}}_{f, 0}=\frac{1}{N} \sum_{k=1}^{N} I_{F}\left(\boldsymbol{x}^{(k)}, \boldsymbol{y}^{(k)}\right) \\
\widehat{\mathrm{P}}_{f, \Theta_{i}}\left(\theta_{i}\right)=\frac{1}{N} \sum_{k=1}^{N} I_{F}\left(\boldsymbol{x}^{(k)}, \boldsymbol{y}^{(k)}\right) r_{\Theta_{i}}\left(\theta_{i} \mid \boldsymbol{x}^{(k)}, \boldsymbol{\theta}^{(k)}\right) \\
\widehat{\mathrm{P}}_{f, \boldsymbol{\Theta}_{i j}}\left(\boldsymbol{\theta}_{i j}\right)=\frac{1}{N} \sum_{k=1}^{N} I_{F}\left(\boldsymbol{x}^{(k)}, \boldsymbol{y}^{(k)}\right) r_{\boldsymbol{\Theta}_{i j}}\left(\boldsymbol{\theta}_{i j} \mid \boldsymbol{x}^{(k)}, \boldsymbol{\theta}^{(k)}\right)
\end{gathered}
$$

The NISS estimators of $\mathrm{P}_{f, Y_{i}}\left(y_{i}\right), \mathrm{P}_{f, \mathbf{Y}_{i j}}\left(\boldsymbol{y}_{i j}\right)$ and $\mathrm{P}_{f, \Theta_{i}, Y_{j}}\left(\theta_{i}, y_{j}\right)$ cannot be established in the similarly way since each $y_{i}$ is an interval input variable other than the distribution parameter of a potential category III model.

By definition, $\mathrm{E}_{\boldsymbol{\theta}, \mathbf{Y}_{-i}}\left[\mathrm{P}_{f}(\boldsymbol{\theta}, \boldsymbol{y})\right]$ can be regarded as a conditional failure probability with the condition that $y_{i}$ is fixed, and $\mathrm{P}_{f, 0}$ is the corresponding unconditional probability. Thus, based on Bayes' rule, the component function $\mathrm{P}_{f, Y_{i}}\left(y_{i}\right)$ can be derived as:

$$
\mathrm{P}_{f, Y_{i}}\left(y_{i}\right)=\mathrm{E}_{\boldsymbol{\Theta}, \mathbf{Y}_{-i}}\left[\mathrm{P}_{f}(\boldsymbol{\theta}, \boldsymbol{y})\right]-\mathrm{P}_{f, 0}=\mathrm{P}_{f, 0} \frac{f_{\mathrm{Y}_{i}}\left(y_{i} \mid F\right)}{f_{\mathrm{Y}_{i}}\left(y_{i}\right)}-\mathrm{P}_{f, 0}=\mathrm{P}_{f, 0} r_{\mathrm{Y}_{i}}\left(y_{i} \mid F\right)
$$

, where $r_{\mathrm{Y}_{i}}\left(y_{i} \mid F\right)=f_{\mathrm{Y}_{i}}\left(y_{i} \mid F\right) / f_{\mathrm{Y}_{i}}\left(y_{i}\right)-1$, and $f_{\mathrm{Y}_{i}}\left(y_{i} \mid F\right)$ indicates PDF of $y_{i}$ conditional on the failure domain $F=\{(\boldsymbol{x}, \boldsymbol{y}): g(\boldsymbol{x}, \boldsymbol{y})<0\}$. The conditional probability density function $f_{Y_{i}}\left(y_{i} \mid F\right)$ is, in general, not known analytically and hence, it can be estimated by any density estimation method, e.g., kernel density estimation, based on the failure samples of $y_{i}$. In other words, this conditional probability density is approximated as $f_{Y_{i}}\left(y_{i} \mid F\right) \approx \hat{f}_{Y_{i}}\left(y_{i} \mid F, S\right)$, where $\hat{f}_{Y_{i}}(\cdot)$ denotes the estimated density, which is deduced based on the sample set $S$. Then, the NISS estimator for $\mathrm{P}_{f, Y_{i}}\left(y_{i}\right)$ can be derived as:

$$
\widehat{\mathrm{P}}_{f, Y_{i}}\left(y_{i}\right)=\widehat{\mathrm{P}}_{f, 0} \hat{r}_{\mathrm{Y}_{i}}\left(y_{i} \mid F, S\right)
$$

where $\hat{r}_{Y_{i}}\left(y_{i} \mid F, S\right)=\hat{f}_{Y_{i}}\left(y_{i} \mid F, S\right) / f_{Y_{i}}\left(y_{i}\right)-1$. Similarly, the NISS estimator of $\mathrm{P}_{f, \mathbf{Y}_{i j}}\left(\boldsymbol{y}_{i j}\right)$ can be derived as:

$$
\widehat{\mathrm{P}}_{f, \mathbf{Y}_{i j}}\left(\boldsymbol{y}_{i j}\right)=\widehat{\mathrm{P}}_{f, 0} \hat{r}_{\mathbf{Y}_{i j}}\left(\boldsymbol{y}_{i j} \mid F, S\right)
$$

where $\hat{r}_{\mathrm{Y}_{i j}}\left(\boldsymbol{y}_{i j} \mid F, S\right)=\hat{f}_{\mathrm{Y}_{i j}}\left(\boldsymbol{y}_{i j} \mid F, S\right) / f_{\mathrm{Y}_{i j}}\left(\boldsymbol{y}_{i j}\right)-\hat{f}_{\mathrm{Y}_{i}}\left(y_{i} \mid F, S\right) / f_{\mathrm{Y}_{i}}\left(y_{i}\right)-\hat{f}_{\mathrm{Y}_{j}}\left(y_{j} \mid F, S\right) / f_{\mathrm{Y}_{j}}\left(y_{j}\right)+1$, and 
$\hat{f}_{\mathrm{Y}_{i j}}\left(\boldsymbol{y}_{i j} \mid F, S\right)$ is the conditional joint PDF of $\boldsymbol{y}_{i j}$ estimated from the sample set $S$, which will be discussed in the next subsection.

Next we derive the NISS estimators for the second-order component function $\mathrm{P}_{f, \Theta_{i}, Y_{j}}\left(\theta_{i}, y_{j}\right)$. By definition, $\mathrm{E}_{\boldsymbol{\Theta}_{-i}, \mathbf{Y}_{-j}}\left[\mathrm{P}_{f}(\boldsymbol{\theta}, \boldsymbol{y})\right]$ can also be regarded as the conditional failure probability with the condition that $y_{j}$ is fixed, and based on the Bayes rule, it can be further derived as:

$$
\mathrm{E}_{\boldsymbol{\Theta}_{-i}, \mathbf{Y}_{-j}}\left[\mathrm{P}_{f}(\boldsymbol{\theta}, \boldsymbol{y})\right]=\mathrm{E}_{\boldsymbol{\Theta}_{-i}, \mathbf{Y}}\left[\mathrm{P}_{f}(\boldsymbol{\theta}, \boldsymbol{y})\right] \frac{f_{\mathrm{Y}_{i}}\left(y_{i} \mid F\right)}{f_{\mathrm{Y}_{i}}\left(y_{i}\right)}=\left[\mathrm{P}_{f, \Theta_{i}}\left(\theta_{i}\right)+\mathrm{P}_{f, 0}\right] \frac{f_{\mathrm{Y}_{i}}\left(y_{i} \mid F\right)}{f_{\mathrm{Y}_{i}}\left(y_{i}\right)}
$$

Thus, $\mathrm{P}_{f, \Theta_{i}, \mathrm{Y}_{j}}\left(\theta_{i}, y_{j}\right)$ is derived as:

$$
\mathrm{P}_{f, \Theta_{i}, \mathrm{Y}_{j}}\left(\theta_{i}, y_{j}\right)=\left[\mathrm{P}_{f, \Theta_{i}}\left(\theta_{i}\right)+\mathrm{P}_{f, 0}\right] r_{\mathrm{Y}_{i}}\left(y_{i} \mid F\right)-\mathrm{P}_{f, \mathrm{Y}_{j}}\left(y_{j}\right)
$$

, and its NISS estimator can be derived as:

$$
\widehat{\mathrm{P}}_{f, \Theta_{i}, \mathrm{Y}_{j}}\left(\theta_{i}, y_{j}\right)=\left[\widehat{\mathrm{P}}_{f, \Theta_{i}}\left(\theta_{i}\right)+\widehat{\mathrm{P}}_{f, 0}\right] \hat{\mathrm{r}}_{\mathrm{Y}_{i}}\left(y_{i} \mid F, S\right)-\widehat{\mathrm{P}}_{f, \mathrm{Y}_{j}}\left(y_{j}\right)
$$

Till now, we have got the NISS estimators for all the first- and second-order component functions based on only one set of joint samples as well as their response values. Thus, the total number of required $g$-function calls is $N$. Naturally, the above generalized NISS procedure owns all the advantages of the classical global NISS procedure. The utilization of Bayes rule for the above generalization is partly inspired by Ref. [28], in which the Bayes rule was utilized for estimating the global reliability sensitivity indices developed by the second author in Ref. [29]. Similarly, the idea of applying Bayes rule for deducing the dependence of the failure probability with respect to a parameter has been explored in Refs. [30] and [31].

The truncation errors as well as the relative importance of each component can be assessed by the Sobol' indices computed from the NISS estimators of the respective component functions, and we don't repeat it for simplicity. It is important to note that the Sobol' indices associated with the uncertain parameters of category II actually stem from the auxiliary probability distributions and hence, are interpreted as representative of the overall impact of those uncertain parameters on the response of interest. Such clarification is important from a theoretical point of view, as Sobol' indices are defined for random variables (and not uncertain parameters of category II). The statistical error of each NISS estimator can be assessed by deriving their respective variance, and the details can be found in Refs. [21] and [22]. In this paper, we implement the NISS method with bootstrap scheme so that the variance of each estimator can be computed in a different way. The procedure of performing the bootstrap for NISS is given as follows.

Step 1: Generate a joint sample set $S=\left\{\left(\boldsymbol{x}^{(\boldsymbol{k})}, \boldsymbol{\theta}^{(\boldsymbol{k})}, \boldsymbol{y}^{(\boldsymbol{k})}\right)\right\}$ of size $N$, and compute the corresponding response values $g^{(k)}$.

Step 2: Randomly generate a new sample set of size $N$ from $\left\{\left(\boldsymbol{x}^{(\boldsymbol{k})}, \boldsymbol{\theta}^{(\boldsymbol{k})}, \boldsymbol{y}^{(\boldsymbol{k})}, \boldsymbol{g}^{(k)}\right)\right\}_{k=1,2, \cdots, N}$ with replacement, and estimate all the component functions as well as their respective Sobol' indices based on this new sample set.

Step 3: Repeat Step 2 for $n_{b}$ (e.g., 50) times so that we can obtain $n_{b}$ estimates for each component function and each Sobol' index, and then compute the mean values and variances for each component function and the corresponding Sobol' index based on their $n_{b}$ estimates.

With the mean value and variance of each component function, we can easily compute the standard 
deviation (STD) and confidence interval of each component function and sensitivity index, based on the fact that each estimator following Gaussian distribution.

With the above procedure, we can synthesize the failure probability function by adding all the influential component functions, and also estimate the confidence bounds of $\mathrm{P}_{f}(\boldsymbol{\theta}, \boldsymbol{y})$ based on the STDs. Third and higher order component functions as well as their corresponding sensitivity indices can be similarly estimated without calling the $g$-function any more. Based on the synthesized failure probability function $\widehat{\mathrm{P}}_{f}(\boldsymbol{\theta}, \boldsymbol{y})$ or the confidence interval of $\mathrm{P}_{f}(\boldsymbol{\theta}, \boldsymbol{y})$, one can estimate the bounds of the failure probability. While the mean estimate $\widehat{\mathrm{P}}_{f}(\boldsymbol{\theta}, \boldsymbol{y})$ is used, the bounds may be either underestimated or overestimated due to the statistical error of $\widehat{\mathrm{P}}_{f}(\boldsymbol{\theta}, \boldsymbol{y})$. However, the effects of the statistical errors on the bounds can be easily assessed since the statistical error of $\widehat{\mathrm{P}}_{f}$ at any fixed location of $(\boldsymbol{\theta}, \boldsymbol{y})$ can be computed. Besides, we can also estimate the lower bound of $\mathrm{P}_{f}$ based on the lower bound of the confidence interval of $\mathrm{P}_{f}(\theta, y)$, and use the upper bound of the confidence interval to estimate the upper bound of $\mathrm{P}_{f}$. With this strategy, the generated bounds include the real bounds with specific level of confidence. For both strategies, the bounds are estimated by numerical optimization procedure. If $\mathrm{P}_{f}(\theta, y)$ is proven by the Sobol' indices to be additive, then only one-dimensional optimization problems need to be solved; while $\mathrm{P}_{f}(\theta, y)$ is also governed by low order interaction terms, then commonly only low-dimensional optimization problems need to be solved. This feature can largely improve the global convergence for estimating bounds.

The auxiliary distribution is one of the key setting for implementing NISS. Theoretically, we can use any types of probability distribution, and this will not affect the formulation of the failure probability function as well as the failure probability bounds, but they do affect the RS-HDMR decomposition in Eq. (10) as well as the distribution of Monte Carlo samples. For reliability analysis, it also affects the number of samples included in the failure domain. We will discuss carefully in the first test example the influence of the different types of auxiliary distribution.

The detailed procedure of NISS is then summarized in Figure 2. In this diagram, the statistical error for the estimate of each component function is assessed by STD in the "Estimation" step, and the truncation error of the synthesized estimation of $\mathrm{P}_{f}(\boldsymbol{\theta}, \boldsymbol{y})$ is measured by sensitivity indices in the "Products" step. 


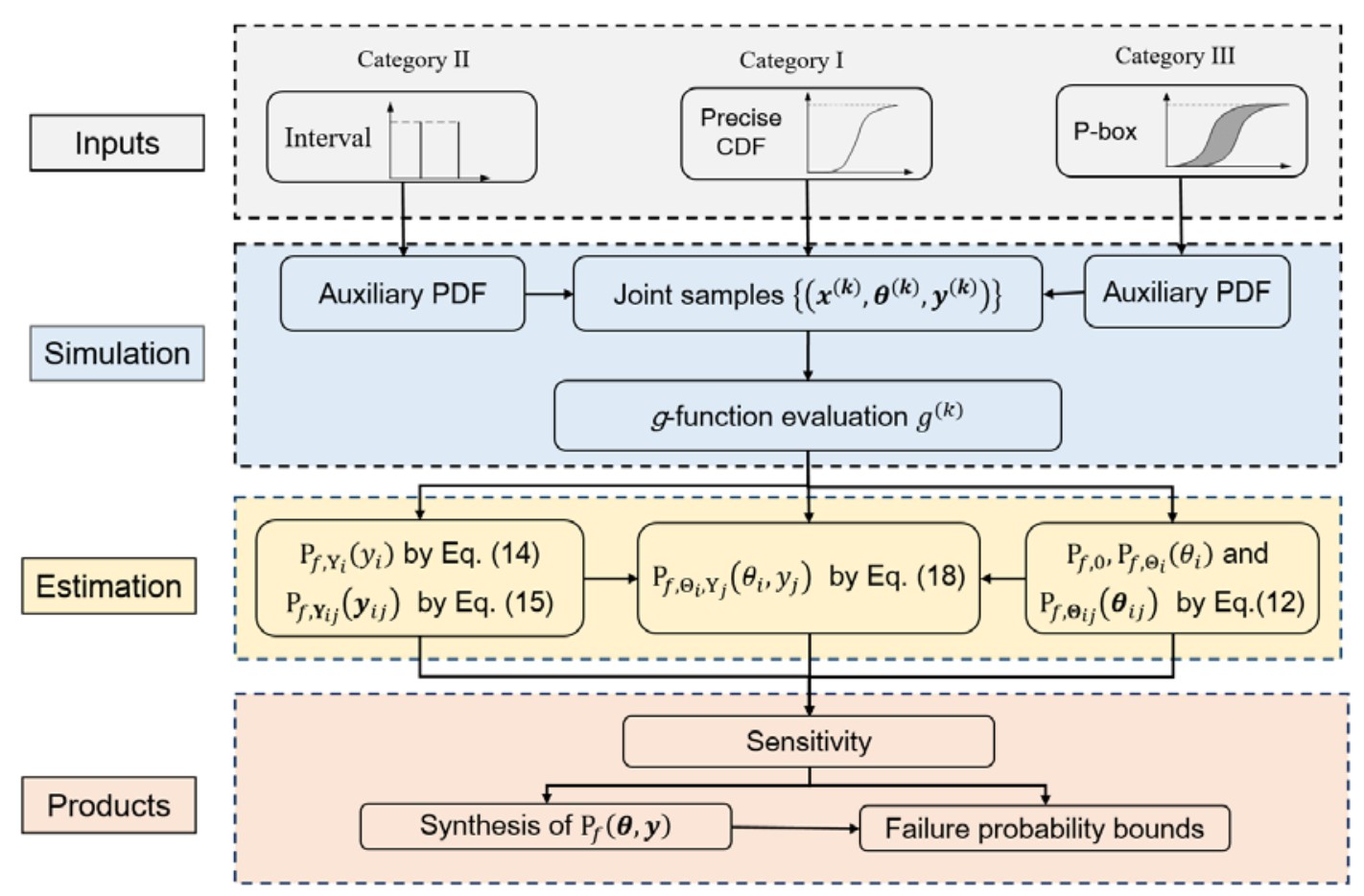

Figure 2. Diagram of the generalized NISS procedure

Although the above NISS procedure needs only one set of $g$-function calls, it is still computational expensive for rare event analysis. However, the computational burden can be largely relieved by injecting subset simulation and/or active learning procedure into the NISS framework, and by implementing it in a parallel scheme. One can refer to Ref. [22] for the details of injection of subset simulation into the NISS framework for estimating the component functions of $\boldsymbol{\theta}$. This strategy can also be extended for estimating the component functions of $\boldsymbol{y}$, as well as their interaction component functions with $\boldsymbol{\theta}$. This extension involves performing subset simulation, in the joint space of $(\boldsymbol{x}, \boldsymbol{\theta}, \boldsymbol{y})$, for estimating $\mathrm{P}_{f, 0}$, and then estimate the conditional density $f_{\mathrm{Y}_{i}}\left(y_{i} \mid F\right)$ based on the failure samples generated with Markov Chain Monte Carlo (MCMC), so as to estimate the component function $\mathrm{P}_{f, \mathrm{Y}_{i}}\left(y_{i}\right)$ by Eq. (13). Further, the active learning procedure can be injected into the above Monte Carlo simulation or subset simulation based NISS framework, and with this improvement, the computational cost can be largely reduced (see Ref. [22] for details).

One should also note that NISS in its current form is not applicable for interval analysis. In traditional interval analysis, only category II inputs are involved, and the target is to estimate the bounds of model response. However, for implementing NISS, it is required at least one category I or category III input is involved, that is, the model inputs must deliver probability distribution information, and the target is to estimate the bounds of probabilistic responses (e.g., failure probability and response distribution function), instead of the bounds of model response.

\subsection{Conditional density estimations}

As can be seen from Eq. (14) and Eq. (15), for interval variables, the performance of the estimators of the corresponding component functions depends on the estimation of the conditional PDFs $f_{\mathrm{Y}_{i}}\left(y_{i} \mid F\right)$ 
and $f_{\mathrm{Y}_{i j}}\left(\boldsymbol{y}_{i j} \mid F\right)$. Thus, in this subsection, we give some useful discussion on the estimations of these two conditional PDF. As has been interpreted in subsection 3.2, both conditional PDFs are estimated based on the failure sample of the interval variables. For univariate PDF $f_{\mathrm{Y}_{i}}\left(y_{i} \mid F\right)$, many non-parametric density estimation procedures have been developed, and in this paper, we suggest using the kernel density estimation (KDE) in Ref. [32] or the function 'ksdensity' in Matlab.

For bivariate joint PDF $f_{\mathrm{Y}_{i j}}\left(\boldsymbol{y}_{i j} \mid F\right)$, we can still use the 2-dimensional KDE developed in Ref. [32] to implement the method. Besides, in many situation of practical applications, the conditional samples of two interval variables may be independent with each other, thus before estimating $f_{\mathrm{Y}_{i j}}\left(\boldsymbol{y}_{i j} \mid F\right)$, we can also perform a hypothesis test on the dependence based on the failure samples of $y_{i}$ and $y_{j}$. In section 7 of our review paper [25], several hypothesis test techniques on variable dependencies have been introduced, and one can refer to this review for more details. If $y_{i}$ and $y_{j}$ are independent, then $f_{\mathrm{Y}_{i j}}\left(\boldsymbol{y}_{i j} \mid F\right)=f_{\mathrm{Y}_{i}}\left(y_{i} \mid F\right) f_{\mathrm{Y}_{j}}\left(y_{j} \mid F\right)$, and the ratio function in Eq. (15) can be further derived as $r_{\mathbf{Y}_{i j}}\left(\boldsymbol{y}_{i j} \mid F\right)=r_{\mathrm{Y}_{i}}\left(y_{i} \mid F\right) r_{\mathrm{Y}_{j}}\left(y_{j} \mid F\right)$, indicating that the second-order component function of $y_{i}$ and $y_{j}$ can be derived based on their respective first-order component functions. If the samples of $y_{i}$ and $y_{j}$ are not independent, one can also use the Copula transformation which reads [33]:

$$
f_{\mathrm{Y}_{i j}}\left(\boldsymbol{y}_{i j} \mid F\right)=c_{i j}\left(u_{i}, u_{j}\right) f_{\mathrm{Y}_{i}}\left(y_{i} \mid F\right) f_{\mathrm{Y}_{j}}\left(y_{j} \mid F\right)
$$

to estimate the joint PDF $f_{\mathrm{Y}_{i j}}\left(\boldsymbol{y}_{i j} \mid F\right)$, where $c_{i j}\left(u_{i}, u_{j}\right)$ is the Copula density function of $y_{i}$ and $y_{j}$, which can be estimated by parametric Copula (e.g., Clayton, Frank, or Gumbel) combined with any distribution parameter estimation procedures, based on the failure samples of $y_{i}$ and $y_{j}$.

\section{Test examples and applications}

\subsection{A toy test example}

Consider a toy example with the following limit state function:

$$
g(\boldsymbol{x}, y)=y^{3} / 3+x_{1}^{2} / 2+x_{2}+1
$$

where $y$ is an interval variable with support $[0,1] ; x_{1}$ is a Gaussian random variable characterized by a $p$-box model, of which the support of the mean parameter $\mu_{1}$ is $[-1,1]$, and the support of STD $\sigma_{1}$ is $[0.8,1.2] ; x_{2}$ is a standard Gaussian random variable with zero mean and unit STD. With the above assumption, the epistemic uncertainties of input variables are characterized by the intervals $y \in[0,1]$, $\mu_{1} \in[-1,1]$ and $\sigma_{1} \in[0.8,1.2]$, while the aleatory uncertainties are characterized by the probability distributions of $x_{1}$ and $x_{2}$. The purpose of analysis is to estimate the failure probability function w.r.t. the three epistemic parameters $y, \mu_{1}$ and $\sigma_{1}$.

For implementing NISS, we need first to specify the auxiliary probability distribution. Theoretically, the type of auxiliary distribution will not affect the failure probability function $\mathrm{P}_{f}\left(y, \mu_{1}, \sigma_{1}\right)$ and the bounds of failure probability, however, it may affect the formulation of each component function. As illustrated in subsection 3.2, we can simply assume that $y$ follows uniform distribution between 0 and 1. However, it is found that, with this assumption, the end regions are not sufficiently well represented, especially the left one of the first-order component function $\mathrm{P}_{f, y}$. This is due to the lack of samples in the left end region. Here three kinds of auxiliary distribution are introduced for $y$, each of which has support $[-0.2,1.2]$. The first one is uniform distribution, and it is abbreviated as "Unif". The second 
one is truncated normal distribution with mean 0.5 and STD 0.35 , and is denoted by “Trun-Norm”. The third one is U-quadratic distribution with density function:

$$
f_{\mathrm{Y}}(y)=\frac{450}{343}(y-0.5)^{2}+0.5
$$

The density functions of three auxiliary distributions are shown in figure 3 . The auxiliary distribution of $\mu_{1}$ and $\sigma_{1}$ are both set to be uniform. Then we set $N=1 \mathrm{e} 5$ and $n_{b}=20$, and generate the joint sample set using Latin hypercube sampling.

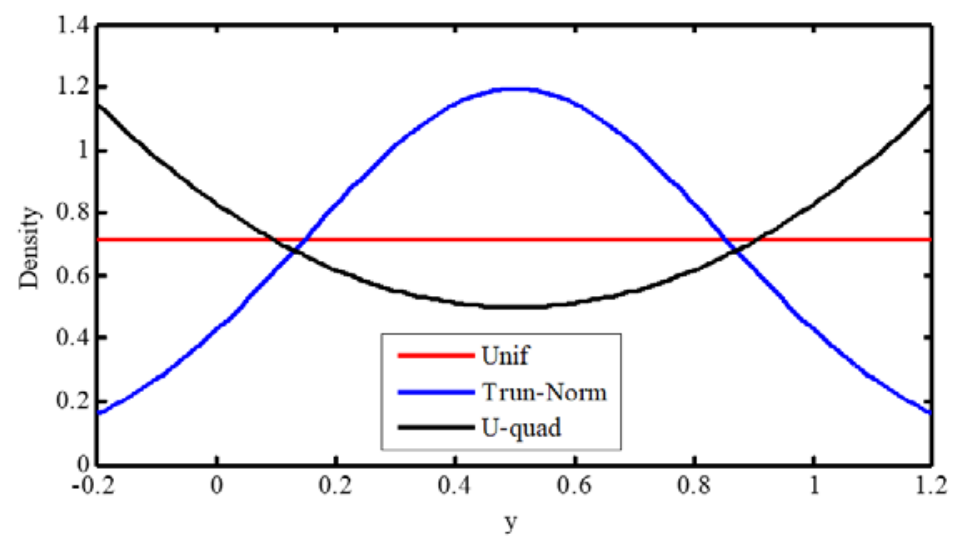

Figure 3 Density functions of the three auxiliary distributions of $y$.

The NISS procedure is implemented with Bootstrap scheme, and the results of the constant HDMR component as well as the first- and second- order Sobol' indices corresponding to each of the three auxiliary distribution are displayed in Table 1, together with the STDs for indicating the convergence of the estimates. As can be seen, for each of the three auxiliary distributions, the relative contribution of each component function is quite similar, and the interaction effects of the three epistemic parameters are quite small. This indicates that the first-order HDMR decomposition provides good approximation for the failure probability function. It can also be seen that, among the three epistemic parameters, $y$ is the most important one, and then $\mu_{1}$ and $\sigma_{1}$, indicating that reducing the epistemic uncertainty of $y$ leads to the most reduction of the epistemic uncertainty of failure probability. This information is quite important for further collecting information on input variables.

Table 1. NISS results of the toy example, where the superscripts indicates the STDs of estimates

\begin{tabular}{|c|c|c|c|c|c|c|c|c|}
\hline $\begin{array}{c}\text { Distribution } \\
\text { type }\end{array}$ & $\mathrm{P}_{f, 0}$ & $\mathrm{~S}_{y}$ & $\mathrm{~S}_{\mu 1}$ & $\mathrm{~S}_{\sigma 1}$ & $\mathrm{~S}_{y \mu 1}$ & $\mathrm{~S}_{y \sigma 1}$ & $\mathrm{~S}_{\mu 1 \sigma 1}$ & $\begin{array}{c}\text { Bounds of } \\
\mathrm{P}_{f}\end{array}$ \\
\hline Unif & $.071^{(8.8 \mathrm{e}-4)}$ & $.695^{(1.9 \mathrm{e}-2)}$ & $.2072^{(1.4 \mathrm{e}-2)}$ & $.0793^{(5.1 \mathrm{e}-3)}$ & $.0068^{(2 \mathrm{e}-4)}$ & $.0026^{(1 \mathrm{e}-4)}$ & $.0091^{(7 \mathrm{e}-4)}$ & {$[.039, .113]$} \\
\hline Trun-Norm & $.075^{(8.7 \mathrm{e}-4)}$ & $.689^{(1.6 \mathrm{e}-2)}$ & $.1983^{(1.1 \mathrm{e}-2)}$ & $.0746^{(5.2 \mathrm{e}-3)}$ & $.0351^{(1 \mathrm{e}-3)}$ & $.0002^{(1 \mathrm{e}-6)}$ & $.0033^{(2 \mathrm{e}-4)}$ & {$[.036, .109]$} \\
\hline U-quad & $.070^{(7.0 \mathrm{e}-4)}$ & $.711^{(2.0 \mathrm{e}-2)}$ & $.179^{(1.2 \mathrm{e}-2)}$ & $.0655^{(4.9 \mathrm{e}-3)}$ & $.0109^{(8 \mathrm{e}-4)}$ & $.0072^{(3 \mathrm{e}-4)}$ & $.0014^{(1 \mathrm{e}-4)}$ & {$[.037, .102]$} \\
\hline
\end{tabular}

The first-order component functions for each kind of auxiliary distribution are estimated by the NISS estimators and the Bootstrap procedure, and the estimations as well as the $95.45 \%$ confidence intervals are shown in Figure 4, together with the reference solutions computed by double-loop crude MCS procedure with $10^{5}$ samples in each iteration. For the three auxiliary distributions, the resulted first-order 
component of $y$ shows very small difference. It is also seen that the estimates of all the three component functions computed by the NISS procedure match well with their reference solutions, and all the three confidence intervals are tight enough. Thus, all the three first-order component functions are effectively estimated by NISS. It is also shown in the first line of Figure 4 that, comparably, around the point $y=0$, the estimation error of $\mathrm{P}_{f, y}$ is a little bit larger for each kind of auxiliary distribution. This can be improved by setting the lower bound of the auxiliary distribution of $y$ a smaller value, say -0.5. Such results are not shown here for the sake of brevity.

Although the second-order component functions are not very influential, we still display their estimates as well as the 95.45\% confidence intervals in Figure 5. As can be seen, all the three component functions are accurately estimated, and for each kind of auxiliary distribution, the second-order component functions are quite similar. One can also find that the ranges of the variations of the three second-order component functions are smaller than those of the first-order component functions. Based on the synthesized mean estimate of $\mathrm{P}_{f}\left(y, \mu_{1}, \sigma_{1}\right)$, the bounds of failure probability is computed by genetic algorithm for each auxiliary distribution, and the results are listed in the last column of Table 1. As can be seen, the bounds generated by the three auxiliary distributions match well.

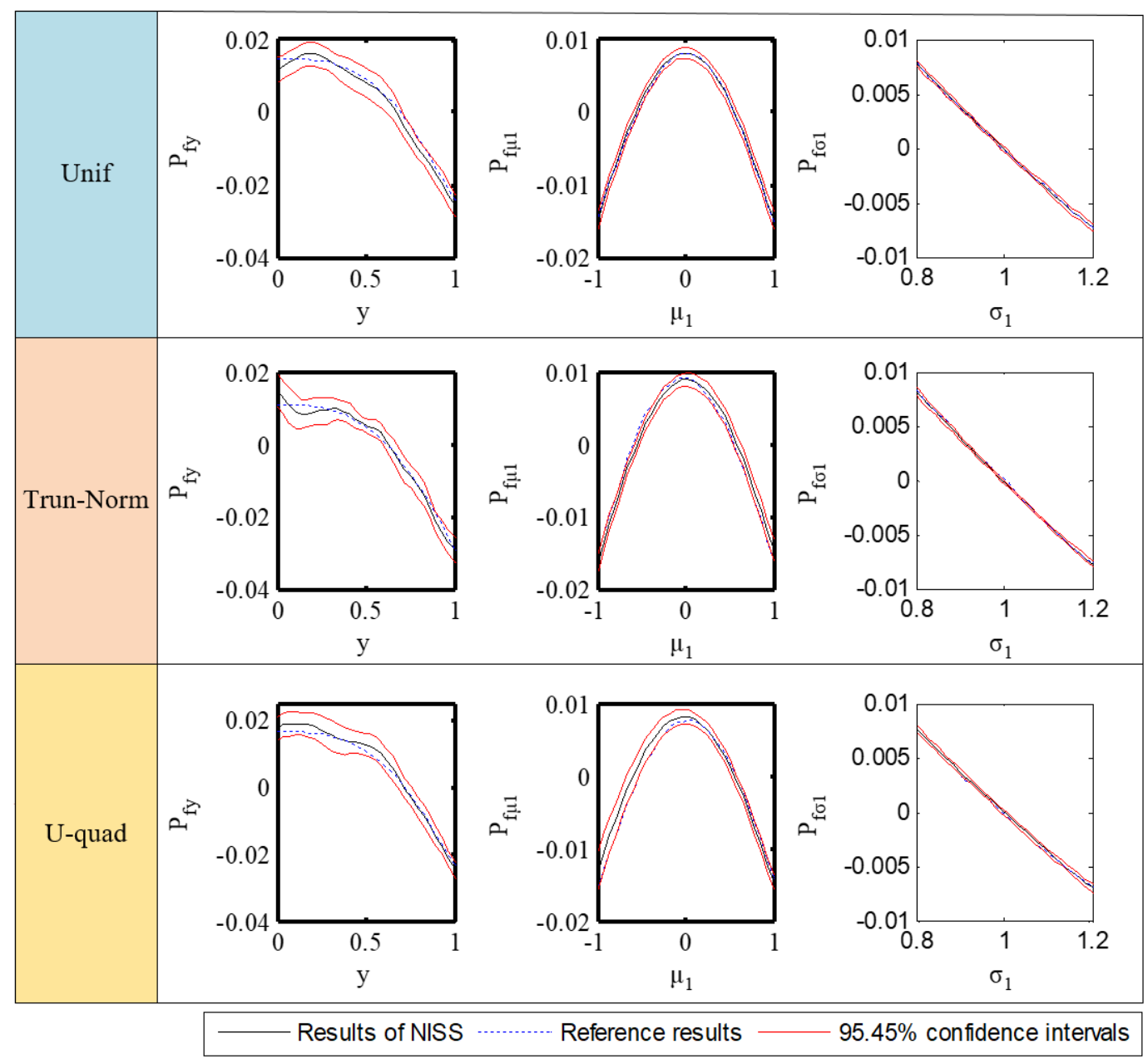

Figure 4. First-order component functions generated based on the three kinds of auxiliary distributions. 


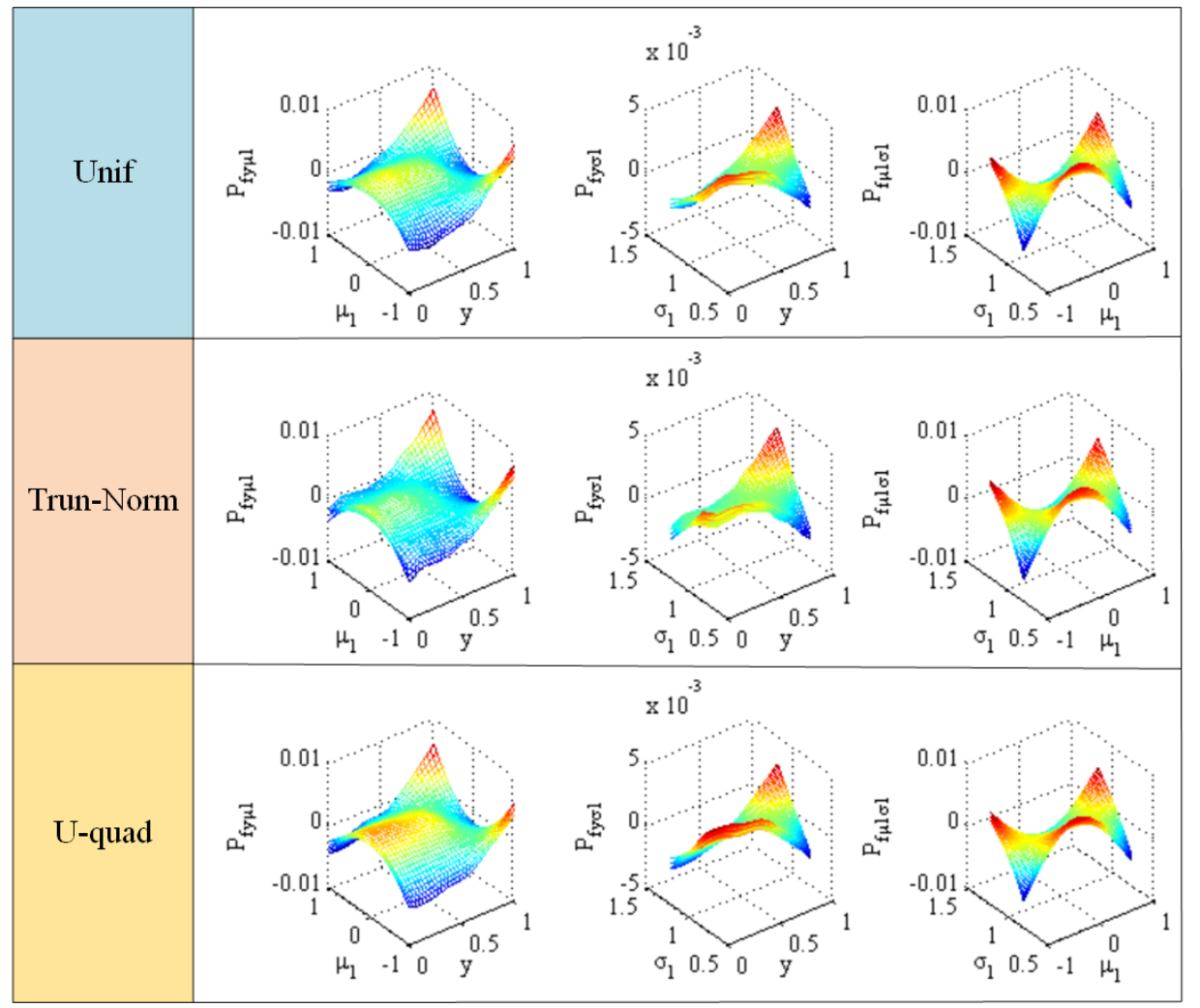

Figure 5. Second-order component functions of the toy example, where the in-between the surfaces indicate the mean estimates, and the other two surfaces indicate the $95.45 \%$ confidence intervals.

\subsection{The NASA Langley multidisciplinary UQ challenge problem}

The NASA Langley UQ challenge problem, released in 2014, describes a real-world aeronautics application. The simulation model aims at simulating the dynamics of remotely operated twin-jet aircraft called Generic Transport Model. One can refer to Ref. [5] for more details on the description of the simulation model. This challenge problem has been dealt with by many researchers (e.g., see Ref. [12][34][35] for details). The challenge problem consists of five subproblems, where the uncertainty propagation and reliability analysis are both important parts. The problem statement of the multidisciplinary reliability analysis is described in Figure 6. The problem is divided into three parts. The first part comprises five fixed discipline analysis, where each of the former four discipline analysis is characterized by a simulation model $h_{i}(\cdot)$ with five input variables, and for the fifth discipline analysis, the response equals the univariate input variable. The response of each discipline analysis is independent with that of the other discipline analysis. The second part is the cross-discipline analysis. This analysis involves eight failure modes, each of which is characterized by a limit state function $g_{i}(\cdot)$ with the five response variables of the fixed discipline analysis as inputs. Thus, the responses of the eight limit state functions are not independent. The third part is the reliability analysis. The eight failure modes are in series, which means that the failure of any mode results in the failure of the whole system. Thus, a composite limit state function $\omega(\cdot, \cdot)$ is defined as the maximum of the eight limit state functions. In Figure 5, the vector $\boldsymbol{d}$ indicates the design variable in the fifth challenge problem (robust design), and in the reliability analysis setting, it is fixed at a pre-specified point $\boldsymbol{d}_{\text {baseline }}$. Thus, the failure probability is defined as the probability of $\omega$ being larger than zero.

Based on the above description, the inputs variables are in fact the 21 input variables $p_{1} \sim p_{21}$ of the 
fixed discipline analysis, and their settings are listed in Table 2. In the first released version of the problem, these 21 inputs variables are grouped into three categories depending on their characterization models. Category I indicates the precise random variables with only aleatory uncertainty. Category II represents the interval variables with only epistemic uncertainty. Category III implies the imprecise random variables with mixed aleatory and epistemic uncertainties. In this paper, there are two main differences of the variable setting with the initial released version. The first difference is on the five inputs of the first discipline analysis. In the initial setting, the categories of the five input variables are the same as those set in Table 2, but the bounds of epistemic intervals are much larger. In the first released version, the first discipline analysis is used for the subproblem of "model updating”, and it is required that the other subproblems should be solved based on the results of this subproblem. Thus, instead of using the initial setting, we use the results of model updating in Ref. [34], for the first five input variables, as shown in Table 2. The second difference is on the support of the three interval variables $p_{6}, p_{12}$ and $p_{16}$. In the initial setting, the support of each variable is $[0,1]$, however, this paper, it is changed to $[0.2,0.8]$, as shown in Table 2. The reason is that, the simulation models don't allow the value of each input variable to exceed the bounds $[0,1]$, however, as indicated by the toy example, the support of auxiliary distribution of each interval variable should better be larger than its real support so that better convergence can be obtained around the end point of real bound. One should note that this modification is not due the limitation of the NISS method, but is due to the limit of the setting of this problem. In other real-world applications, the physically allowed interval of one interval variable is commonly wider than the uncertainty support, making it possible to set the support of the auxiliary distribution wider than the uncertainty support. As shown in Table 2, with the above setting, we have 31 epistemic parameters, and they are denoted as $\theta_{1} \sim \theta_{31}$, and then the system failure probability will be a function on these 31 parameters, and the purpose of this example is to estimate this failure probability function as well as the related failure probability bounds. It should be noted that for the sake of compactness, all epistemic parameters in Table 2 are labeled as $\theta_{i}, i=1, \ldots, 31$; nonetheless, parameters $\theta_{3}, \theta_{9}, \theta_{16}$ and $\theta_{23}$ should have been actually labeled as $y_{1}, y_{2}, y_{3}$ and $y_{4}$, respectively.

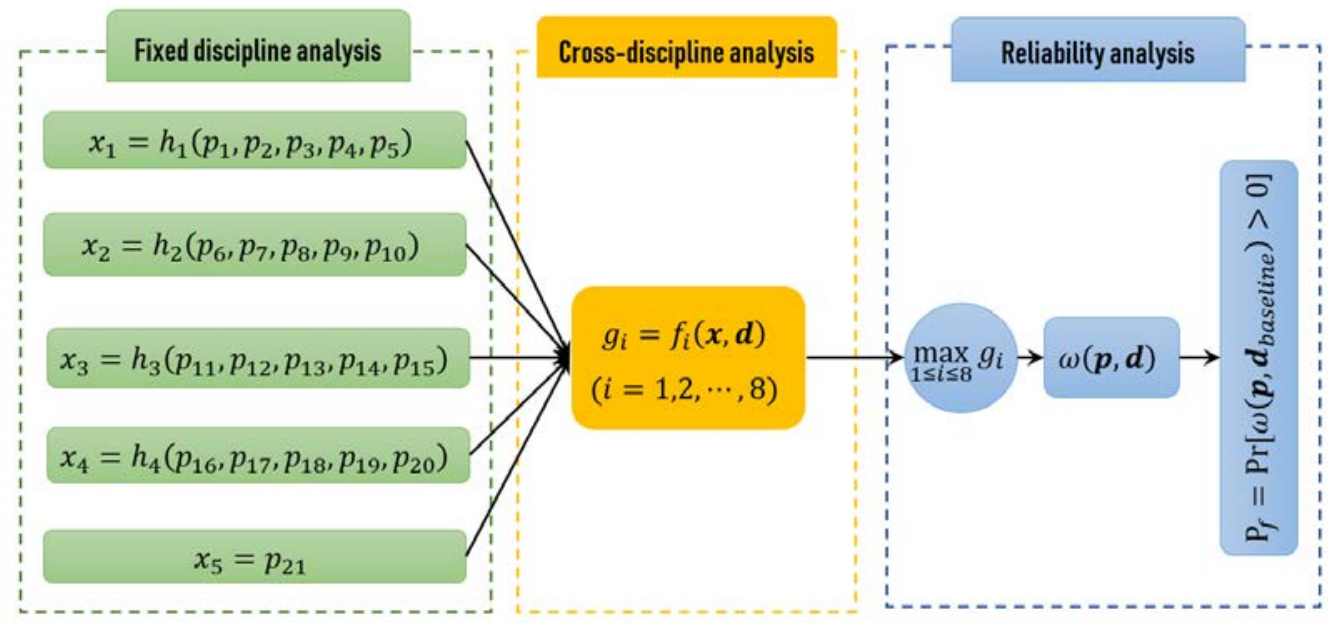

Figure 6. Description of the NASA multidisciplinary UQ challenge problem and the related reliability analysis sub-problem 
Table 2 Uncertain parameters of the NASA UQ challenge problem, where $\theta_{1} \sim \theta_{31}$ indicates the epistemic parameters.

\begin{tabular}{|c|c|c|}
\hline Input variables & Category & Uncertainty model \\
\hline$p_{1}$ & III & $\begin{array}{c}\text { Unimodal Beta, } 0.6783 \leq \theta_{1}=\mu_{1} \leq 0.7097,0.0387 \leq \theta_{2}^{2}= \\
\sigma_{1}^{2} \leq 0.0397\end{array}$ \\
\hline$p_{2}$ & II & Interval, $\theta_{3}=p_{2} \in[0.9399,0.9902]$ \\
\hline$p_{3}$ & I & Uniform, $[0,1]$ \\
\hline$p_{4}, p_{5}$ & III & $\begin{array}{c}\text { Normal, } 3.4493 \leq \theta_{4}=\mu_{4} \leq 4.5812,0.4190 \leq \theta_{5}^{2}=\sigma_{4}^{2} \leq \\
2.7209,-1.5306 \leq \theta_{6}=\mu_{5} \leq-0.9106,0.2157 \leq \theta_{7}^{2}=\sigma_{5}^{2} \leq \\
0.6914,-0.4370 \leq \theta_{8}=\rho \leq 0.7008\end{array}$ \\
\hline$p_{6}$ & II & Interval, $\theta_{9}=p_{6} \in[0.2,0.8]$ \\
\hline$p_{7}$ & III & Beta, $0.982 \leq \theta_{10}=a \leq 3.537,0.619 \leq \theta_{11}=b \leq 1.080$ \\
\hline$p_{8}$ & III & Beta, $7.450 \leq \theta_{12}=a \leq 14.093,4.285 \leq \theta_{13}=b \leq 7.864$ \\
\hline$p_{9}$ & I & Uniform, $[0,1]$ \\
\hline$p_{10}$ & III & Beta, $1.520 \leq \theta_{14}=a \leq 4.513,1.536 \leq \theta_{15}=b \leq 4.750$ \\
\hline$p_{11}$ & I & Uniform, $[0,1]$ \\
\hline$p_{12}$ & II & Interval, $\theta_{16}=p_{12} \in[0.2,0.8]$ \\
\hline$p_{13}$ & III & Beta, $0.412 \leq \theta_{17}=a \leq 0.737,1.000 \leq \theta_{18}=b \leq 2.068$ \\
\hline$p_{14}$ & III & Beta, $0.931 \leq \theta_{19}=a \leq 2.169,1.000 \leq \theta_{20}=b \leq 2.407$ \\
\hline$p_{15}$ & III & Beta, $5.435 \leq \theta_{21}=a \leq 7.095,5.287 \leq \theta_{22}=b \leq 6.945$ \\
\hline$p_{16}$ & II & Interval, $\theta_{23}=p_{16} \in[0.2,0.8]$ \\
\hline$p_{17}$ & III & Beta, $1.060 \leq \theta_{24}=a \leq 1.662,1.000 \leq \theta_{25}=b \leq 1.488$ \\
\hline$p_{18}$ & III & Beta, $1.000 \leq \theta_{26}=a \leq 4.266,0.553 \leq \theta_{27}=b \leq 1.000$ \\
\hline$p_{19}$ & I & Uniform, $[0,1]$ \\
\hline$p_{20}$ & III & Beta, $7.530 \leq \theta_{28}=a \leq 13.492,4.711 \leq \theta_{29}=b \leq 8.148$ \\
\hline$p_{21}$ & III & Beta, $0.421 \leq \theta_{30}=a \leq 1.000,7.772 \leq \theta_{31}=b \leq 29.621$ \\
\hline
\end{tabular}

With the simple random sampling, we generate $5 \times 10^{4}$ joint samples for $\left(p_{1}, \cdots, p_{21}, \theta_{1}, \cdots, \theta_{31}\right)$, and compute the response value of limit state function $\omega\left(\boldsymbol{p}, \boldsymbol{d}_{\text {baseline }}\right)$ for each joint sample, to implement the NISS procedure in a bootstrap manner. The number of bootstrap replication is set to be 30 . Thus the total number of function calls is 5e4. With these samples, the constant RS-HDMR component of the failure probability is computed as 0.2319 with the STD being 0.0017 .

With the same set of samples, the non-normalized first-order Sobol' indices are computed, and the results are shown in Figure 7, together with the STD of each estimate. As can be seen, all the sensitivity indices are robustly estimated. The results show that, among the 31 epistemic parameters, only a small number of them are influential, and the six most influential components are those of $\theta_{16}, \theta_{1}, \theta_{3}, \theta_{23}, \theta_{9}$ and $\theta_{5}$. The summation of the sensitivity indices of the other 25 first-order component functions is smaller than one percent of the summation of the sensitivity indices of these six most influential. Thus, 
we need only to consider the six most influential first-order component function. The sensitivity indices also reveals that, among the 31 epistemic parameters, the epistemic uncertainty of $\theta_{16}$ (i.e., $p_{12}$ ) contributes the most to the epistemic uncertainty of failure probability. Thus, for reducing the bounds of the failure probability, one should collect more information on $p_{12}$. Based on the same set of samples, the six first-order component functions are estimated, and the estimates as well as the $95.45 \%$ confidence intervals are shown in Figure 8. It is shown that the confidence interval of each component function is narrow enough, indicating that each component function is accurately estimated by NISS.

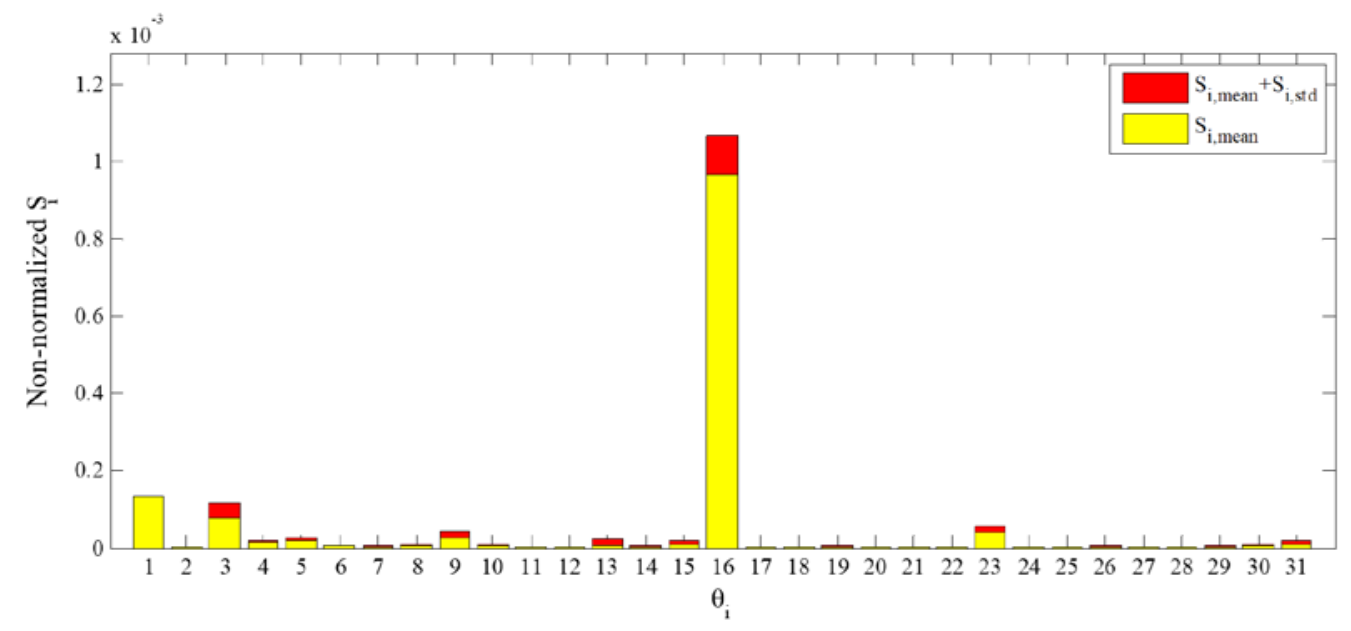

Figure 7. The first-order normalized sensitivity indices computed by NISS procedure
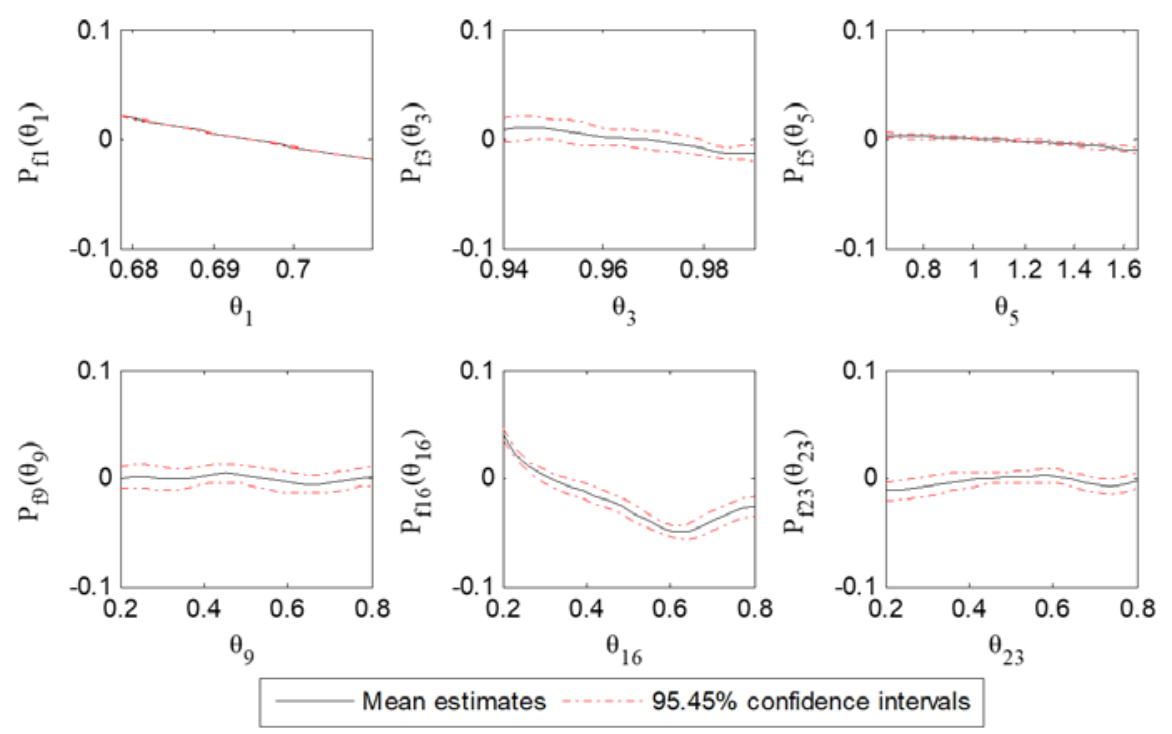

Figure 8. The first-order component functions of the NASA UQ challenge problem

Next, we go into the second-order component functions. As has been discussed in subsection 3.3, for the second-order component function of two interval input variables, we need to estimate their joint PDF based on their samples belonging to the failure domain. We randomly select 1e3 sample points for each pair of interval variables from their failure samples, and transform these data into copula scale by using the function 'ksdensity' in Matlab, and then plot the samples in pair in Figure 9. As can be seen, for each 
pair of interval variables, the sample points are uniformly distributed in the unit square space $[0,1]^{2}$, indicating that each pair of interval variables are independent in the failure domain. Then, based on the discussion in subsection 3.3, the second-order component functions of each pair of interval variables can be easily estimated by their first-order component functions, and we don't need to estimate the joint PDF. The six most influential second-order component functions are then estimated with the same set of samples, and the estimates as well as the $95.45 \%$ confidence intervals are displayed in Figure 10. As can be seen, compared with the first-order component functions, all the second-order component functions can be neglected.

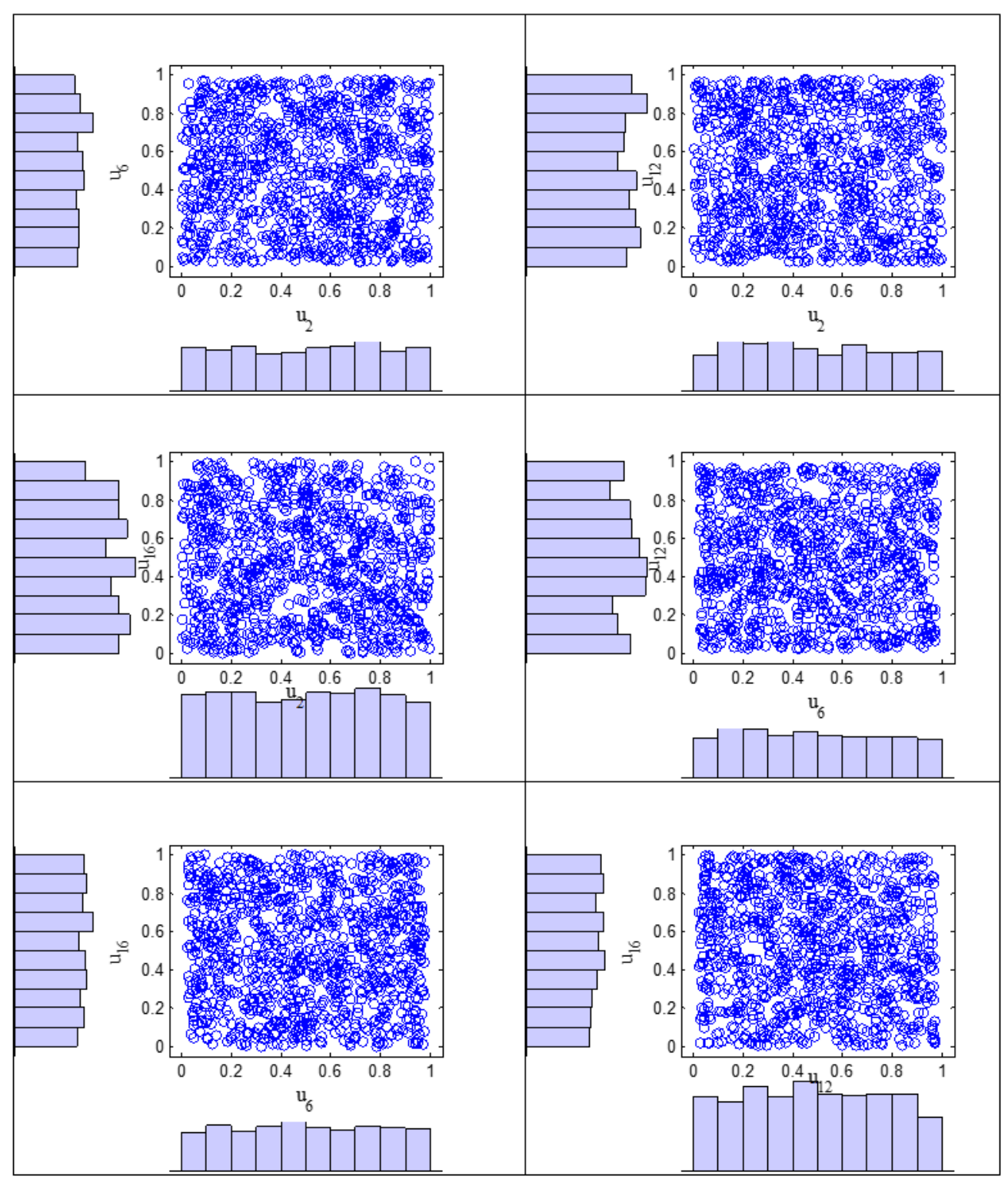

Figure 9 Pairwise scatter and histogram plots of the failure data of the four interval variables $p_{2}, p_{6}$, $p_{12}$ and $p_{16}$ in copula scale (unit square), where the transformation to copula scale is realized by the "ksdensity" function.

Based on mean estimate of the failure probability function synthesized with the six influential first- 
order component functions and the two most influential second-order component functions, the bounds of failure probability are estimated to be [0.1221, 0.3121]. Since the settings of the interval variables are different to the original ones, and the results of model updating of the first discipline vary from paper to paper, thus it not possible to compare the results with the published results in, e.g., Refs. [11] and [35]. To demonstrate the correctness of our result, we also estimate the failure probability bounds by the interval Monte Carlo simulation (IMCS) developed in Ref. [13], which has also been utilized in Ref. [35] for estimating the failure probability bounds of the NASA challenge problem. This method involves a double-loop procedure. In the outer loop, the interval samples are generated for the input variables, while in the inner loop, the interval analysis is performed for generating the bounds of limit state function for each interval sample. The failure probability bounds are then estimated based on the samples of response bounds. Due to the large computational cost, this procedure is implemented in a parallel scheme on a 48core computer station. One should note that, the bounds estimated by IMCS in this example are more conservative since in this method, the parameterized p-box model is by default replaced by a nonparameterized p-box model with the bounds as the parameterized ones. This simplification is necessary in IMCS for generating interval samples in the outer loop. Similar to Ref. [35], 1000 interval samples are generated in the outer loop, and the genetic algorithm is utilized in the inner loop, to implement the IMCS method. The reference bounds are estimated to be [0.055, 0.337], which exactly include the bounds generated by NISS. Based on the above analysis, it should be believed that the bounds computed by NISS are correct.
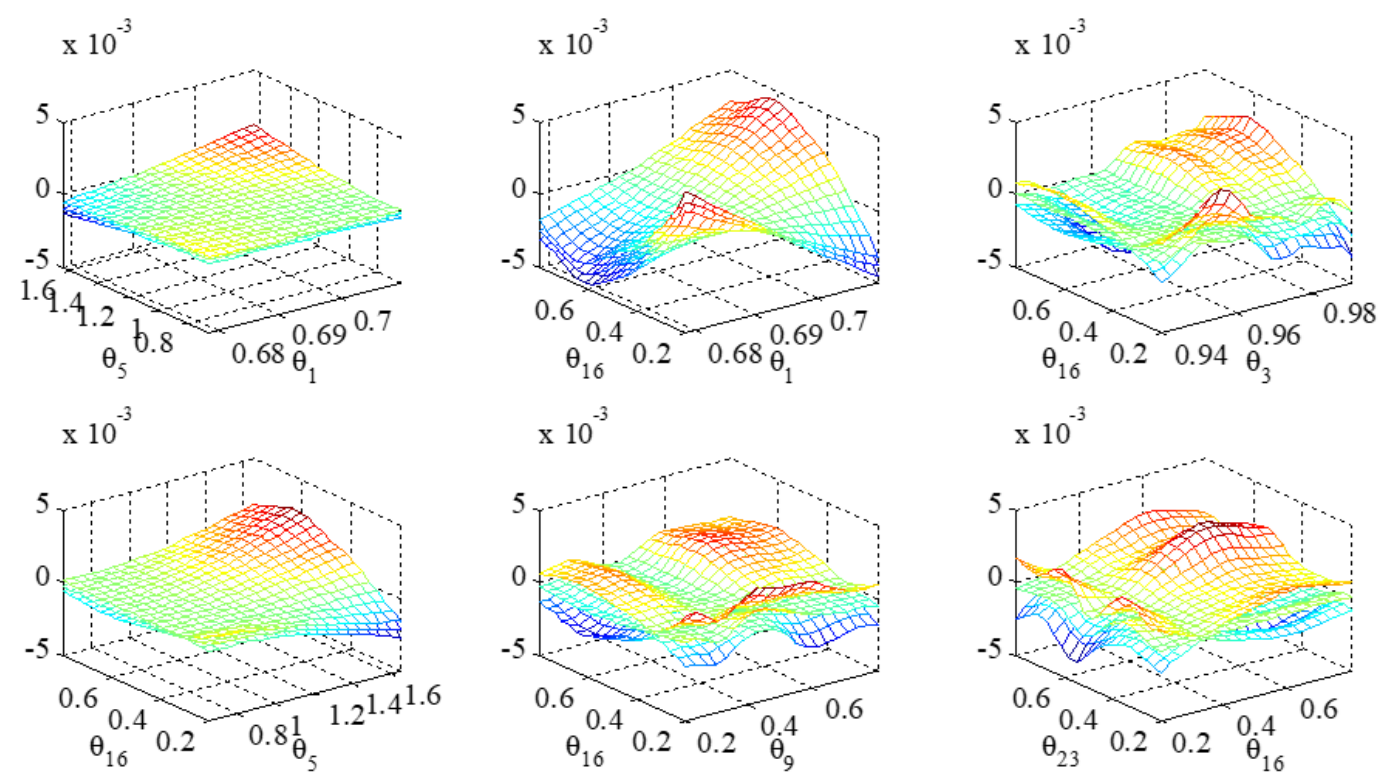

Figure 10. The six most influential second-order component functions

\section{Conclusions and discussions}

This paper has developed a strategy for generalizing the NISS method, recently developed for efficiently propagating the imprecise probability models, to the general case where three categories of characterization models (i.e., precise probability models, non-probabilistic models, and imprecise probability models) are all involved, and specifically, the estimation of failure probability function and 
bounds are exemplified. The truncation errors of estimates are quantified by Sobol' sensitivity indices, which are also found to be useful for measuring the relative importance of the component functions as well as each epistemic parameter. Both the toy test example and the NASA Langley challenge have demonstrated the effectiveness of the proposed method.

The NISS method owns many advantages, and the most appealing one is that only one set of function calls are needed for implementing the whole analysis, and both types of estimation errors are properly addressed without extra computational cost. Being a pure stochastic simulation procedure, it is easy to implement it in a parallel scheme, making it more efficient for large-scale real-world applications. The only limitation of the generalization of the NISS reported here is that, for the component functions of interval variables, there is a need to do non-parametric density estimation, which, for first-order component functions, can be addressed with kernel density estimation, but for higher order component functions, may face challenge. However, the estimation of bivariate density functions can be properly addressed by statistic dependence or parameterized Copula, as has been shown in subsection 3.3 and 4.2.

The success of NISS for high-dimensional problems is supported by the following facts. For most real physic processes, the model behavior is mainly governed by a low-dimensional manifold, and this manifold is mostly governed by individual and/or low-order interaction effects. The introduction of the RS-HDMR decomposition as well as the Sobol' sensitivity indices enables to identify this manifold and the influential effects without extra computational cost. Further, the RS-HDMR decomposition makes it possible to derive NISS estimators for the component functions of the epistemic parameters presented in both category II and category III models.

Open problems still exist. For example, the distribution-free category III models such as nonparametric p-box model are also widely used when the distribution type is not known due to lack of knowledge; the multivariate dependence may exist in category II model due to the natural constraints of model parameters; time-variant and spatial inputs are also commonly in real-world applications. There is a need to extend the method to the above situations. Besides, the uncertainty-based design optimization problem, e.g., the robust optimization subproblem in the NASA Langley UQ challenge problem [11], and the sensitivity analysis under mixed uncertain environment [36], can also be addressed by the NISS method, which will be presented in the future work.

\section{Acknowledgments}

This work is supported by the National Natural Science of China (NSFC 51905430) and the Aerospace Science and Technology Foundation of China. The first author is supported by the program of China Scholarships Council (CSC). The second to fourth authors are all supported by the Alexander von Humboldt Foundation of Germany. The second author is also supported by the Top International University Visiting Program for Outstanding Young Scholars of Northwestern Polytechnical University. The third author acknowledges the support by CONICYT (National Commission for Scientific and Technological Research) under grant number 1180271. The authors also appreciate the two anonymous reviewers for their very useful comments and suggestions.

\section{Reference}


[1] A. Der Kiureghian, O. Ditlevsen, Aleatory or epistemic? Does it matter? Struct. Saf. 31 (2009) 105112

[2] M. Faes, D. Moens, Recent Trends in the Modeling and Quantification of Non-probabilistic Uncertainty, Arch. Comput. Method. E. 2019, https://doi.org/10.1007/s11831-019-09327-x

[3] C.Jiang, R.G. Bi, G.Y. Lu, X. Han, Structural reliability analysis using non-probabilistic convex model, Comput. Method Appl. M. 254(2013) 83-98.

[4] M. Beer, S. Ferson, V. Kreinovich, Imprecise probabilities in engineering analyses, Mech. Syst. Signal Process. 37 (2013) 4-29

[5] L.G. Crespo, S.P. Kenny, D.P. Giesy, The NASA Langley multidisciplinary uncertainty quantification challenge, in: 16th AIAA Non-Deterministic Approaches Conf., 2014, pp. 1-9, https://doi.org/10.2514/6.2014-1347.

[6] J. Li, J. Chen, W. Sun, Y. Peng, Advances of the probability density evolution method for nonlinear stochastic systems, Probabilist. Eng. Mech. 28 (2012) 132-142.

[7] S.K. Au, J.L. Beck, A new adaptive important sampling scheme, Struct. Saf. 21(1999) 135-158.

[8] S.K. Au, J.L. Beck, Estimation of small failure probabilities in high dimensions by subset simulation, Probabilist. Eng. Mech. 16(2001) 263-277.

[9] G.I. Schuëller, H.J. Pradlwarter, P.S. Koutsourelakis, A critical appraisal of reliability estimation procedures for high dimensions, Probabilist. Eng. Mech. 19(2004) 463-473.

[10] P. Wei, F. Liu, C. Tang, Reliability and reliability-based importance analysis of structural systems using multiple response Gaussian process model, Reliab. Eng. Syst. Safe. 175(2018) 183-195.

[11] M. de Angelis, E. Patelli and M. Beer, Advanced Line Sampling for efficient robust reliability analysis, Struct. Saf. 52 (2015) 170-182

[12] N. Pedroni, E. Zio, Hybrid uncertainty and sensitivity analysis of the model of a twin-jet aircraft, J. Aerosp. Inform. Syst. 12(1)(2015) 73-96.

[13] H. Zhang, H. Dai, M. Beer, W. Wang, Structural reliability analysis on the basis of small samples: an interval quasi-Monte Carlo method, Mech. Syst. Signal Process. 37 (2013) 137-151.

[14] W. Cao, H. Zhang, M. Beer, Computing tight bounds of structural reliability under imprecise probabilistic information, Comput. Struct. 208 (2018) 92-104.

[15] S.F. Bi, M. Broggi, P.F. Wei, M. Beer. The Bhattachayya distance: Enriching the P-box in stochastic sensitivity analysis, Mech. Syst. Signal Process. 129(2019): 265-281

[16] W. Gao, D. Wu, K. Gao, X. Chen, F. Tin-Loi, Structural reliability analysis with imprecise random and interval fields, Appl. Math. Model. 55(2018) 49-67.

[17] D. Wu, W. Gao. Hybrid uncertain static analysis with random and interval fields, Comput. Method Appl. M. 315 (2017) 222-246.

[18] J. Feng, D. Wu, W. Gao, G. Li. Hybrid uncertain natural frequency analysis for structures with random and interval fields, Comput. Method Appl. M. 328(2018) 365-389.

[19] P.F. Wei, Z.Z. Lu, J.W. Song, Extended Monte Carlo simulation for parametric global sensitivity analysis and optimization, AIAA J. 52 (2014) 867-878

[20] J. Zhang, M.D. Shields, On the quantification and efficient propagation of imprecise probabilities resulting from small datasets, Mech. Syst. Signal Process. 98 (2018) 465-483. 
[21] P.F. Wei, J.W. Song, S.F. Bi, M. Broggi, M. Beer, Z.F. Yue, Z.Z. Lu, Non-intrusive stochastic analysis with parameterized imprecise probability models: I. Performance estimation, Mech. Syst. Signal Process. 124 (2019) 349-368.

[22] P.F. Wei, J.W. Song, S.F. Bi, M. Broggi, M. Beer, Z.F. Yue, Z.Z. Lu, Non-intrusive stochastic analysis with parameterized imprecise probability models: II. Reliability and rare events analysis, Mech. Syst. Signal Process. 126 (2019) 227-247.

[23] G.Y. Li, S.W. Wang, H. Rabitz, Practical approaches to construct RS-HDMR component functions, J. Phys. Chem. A 106 (2002) 8721-8733.

[24] I.M. Sobol', S. Tarantola, D. Gatelli, S.S. Kucherenko, W. Mauntz, Estimating the approximation error when fixing unessential factors in global sensitivity analysis, Reliab. Eng. Syst. Safe. 92 (2007) 957-960.

[25] P.F. Wei, Z. Z. Lu, J. W. Song, Variable importance analysis: a comprehensive review, Reliab. Eng. Syst. Safe. 142(2015) 399-342

[26] P. Wang, Z.Z. Lu, K.C. Zhang, S.N. Xiao, Z.F. Yue, Copula-based decomposition approach for the derivative-based sensitivity of variance contributions with dependent variables, Reliab. Eng. Syst. Safe. 169(2018) 437-450

[27] J.C. Helton, J.D. Johnson, W.L. Oberkampf, C.J. Sallaberry, Representation of analysis results involving aleatory and epistemic uncertainty, Int. J. Gen. Syst. 39 (2010) 605-646.

[28] W.Y. Yun, Z.Z. Lu, Y. Zhang, X. Jiang, An efficient global reliability sensitivity analysis algorithm based on classification of model output and subset simulation, Struct. Saf. 74(2018) 49-57.

[29] P.F. Wei, Z.Z. Lu, W.R. Hao, J. Feng, B.T. Wang, Efficient sampling methods for global reliability sensitivity analysis, Comput. Phys. Commun. 183(2012) 1728-1743.

[30] S.K. Au. Reliability-Based Design Sensitivity by Efficient Simulation, Comput. Struct. 83(2005) 1048-1061.

[31] J. Ching, Y. Hsieh. Local Estimation of Failure Probability Function and its Confidence Interval with Maximum Entropy Principle, Probabilist. Eng. Mech. 22(2007) 39-49.

[32] Z.I. Botev, J.F. Grotowski, D.P. Kroese, Kernel density estimation via diffusion. Ann. Stat. 38(2010) 2916-2957.

[33] P.F. Wei, Z.Z. Lu, J.W. Song, Moment-Independent Sensitivity Analysis Using Copula, Risk Anal. 34(2014), 210-222.

[34] S.F. Bi, M. Broggi, M. Beer, The role of the Bhattacharyya distance in stochastic model updating, Mech. Syst. Signal Process. 117(2019) 437-452

[35] E. Patelli, D.A. Alvarez, M. Broggi, M.D. Angelis, Uncertainty management in multidisciplinary design of critical safety systems, J. Aerosp. Inform. Syst. 12(1)(2015) 140-169.

[36] P.F. Wei, F.C. Liu, Z.Z. Lu, Z.T. Wang (2018). A probabilistic procedure for quantifying the relative importance of model inputs characterized by second-order probability models. Int. J. Approx. Reason. 98(2018), 78-95. 